\title{
Optimized Halogen Atomic Radii for PBSA Calculations Using Off-Center Point-Charges
}

\author{
Andreia Fortuna ${ }^{\dagger, \ddagger}$ and Paulo J. Costa*,† \\ $\dagger$ †ioISI - Biosystems 83 Integrative Sciences Institute, Faculty of Sciences, University of \\ Lisboa, 1749-016, Lisboa, Portugal \\ $\ddagger$ Research Institute for Medicines (iMed.ULisboa), Faculty of Pharmacy, University of \\ Lisbon, Av. Professor Gama Pinto, 1649-003 Lisbon, Portugal \\ E-mail: pjcosta@ciencias.ulisboa.pt, pjcosta@fc.ul.pt
}

Phone: +351-21-7500196

\begin{abstract}
In force field methods, the usage of off-center point-charges, also called extra-points (EPs), is a common strategy to tackle the anisotropy of the electrostatic potential of covalently-bonded halogens (X), thus allowing the description of halogen bonds (XBs) at the molecular mechanics / molecular dynamics (MM/MD) level. Diverse EP implementations exist in the literature differing on the charge sets and/or the $\mathrm{X}$-EP distances. Poisson-Boltzmann and surface area (PBSA) calculations can be used to obtain solvation free energies $\left(\Delta G_{\text {solv }}\right)$ of small molecules, often to compute binding free energies ( $\left.\Delta G_{\text {bind }}\right)$ at the MM-PBSA level. This method depends, among other parameters, on the empirical assignment of atomic radii (PB radii). Given the multiplicity of off-center point-charges models and the lack of specific PB radii for halogens compatible with such implementations, in this work we assessed the performance of PBSA calculations for the estimation of $\Delta G_{\text {solv }}$ values in water $\left(\Delta G_{\text {hyd }}\right)$, also conducting an optimization of the halogen $\mathrm{PB}$ radii $(\mathrm{Cl}, \mathrm{Br}$, and $\mathrm{I})$ for each $\mathrm{EP}$ model. We
\end{abstract}


not only expand the usage of EP models in the scope of the General AMBER Force Field (GAFF) but also provide the first optimized halogen PB radii in the context of the CHARMM General Force Field (CGenFF), thus contributing to improving the description of halogenated compounds in PBSA calculations.

\section{Introduction}

The determination of binding free energies $\left(\Delta G_{\text {bind }}\right)$ is of extreme importance in computeraided drug discovery ${ }^{1,2}$ and, in order to obtain accurate results, the solvation free energy $\left(\Delta G_{\text {solv }}\right)$ must be properly estimated. Furthermore, $\Delta G_{\text {solv }}$ is by itself an important property in the study and characterization of compounds, therefore, the estimation of ligand hydration free energies, $\Delta G_{\text {hyd }}$, is paramount as most biomolecular processes occur in water. ${ }^{3,4}$ Free energy perturbation (FEP) and thermodynamic integration (TI) methods can be used to calculate both $\Delta G_{\text {bind }}$ and $\Delta G_{\text {solv }},{ }^{5-9}$ but at a cost of being computationally demanding. On the other hand, the molecular mechanics-Poisson-Boltzmann surface area (MM-PBSA) method has the advantage of requiring a much lower computational cost to obtain both quantities, hence, it can be applied to large libraries of compounds. ${ }^{10,11}$ In this method, the hydration (solvation) free energies of the components of the system (e.g. protein-ligand) are estimated using the Poisson-Boltzmann (PB) equation and surface area (SA) calculations, which, among other terms, depends on atomic charges and a set of atomic radii, often called $\mathrm{PB}$ radii, which are used to define the solute $\left(\epsilon_{\text {in }}\right)$ and solvent $\left(\epsilon_{\text {out }}\right)$ regions (Figure 1).

Covalently-bonded halogens $(\mathrm{R}-\mathrm{X}$, with $\mathrm{X}=\mathrm{Cl}, \mathrm{Br}, \mathrm{I})$ possess anisotropic electronic features, showing a positive region in their electrostatic potential, called the $\sigma$-hole, which enables their interaction with a Lewis base (B). ${ }^{12}$ This type of $\mathrm{R}-\mathrm{X} \cdots \mathrm{B}$ interaction, known as halogen bond (XB), ${ }^{13}$ has found application in diverse fields of (bio)chemical sciences. ${ }^{14,15}$ In-

deed, there are numerous examples were XBs play an important role in anion recognition, ${ }^{16,17}$ catalysis, ${ }^{18}$ in protein-ligand ${ }^{19-21}$ and membrane-ligand ${ }^{22}$ systems, these latter cases showing their relevance in medicinal chemistry. In fact, halogenated compounds are strongly present 


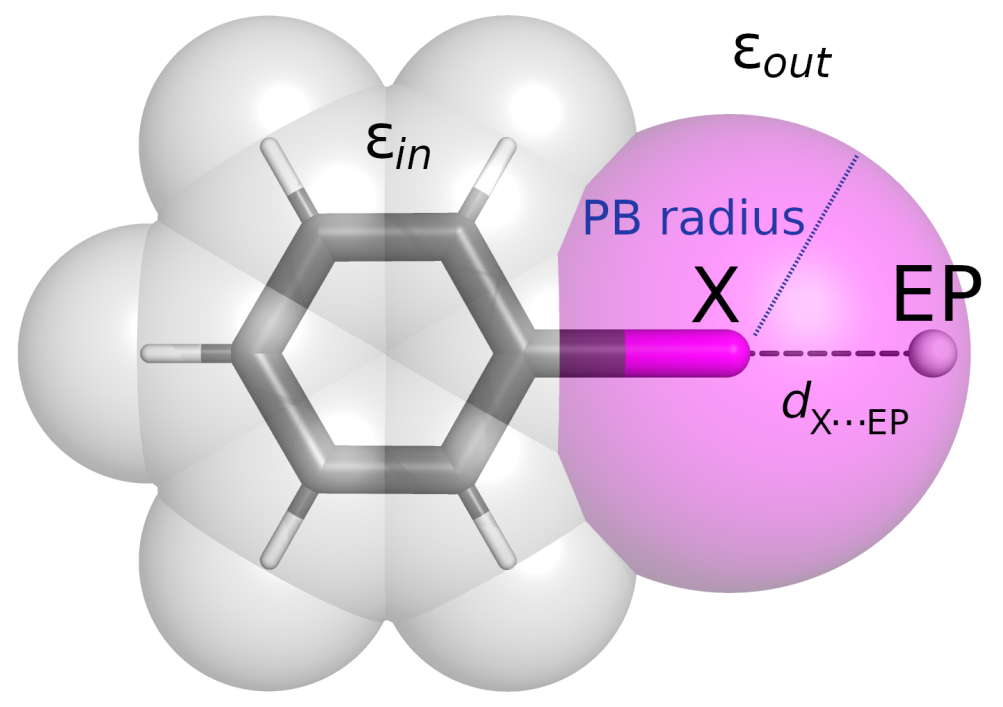

Figure 1: Schematic representation of a halogenated molecule in a PBSA calculation: an offcenter point-charge $(\mathrm{EP})$ is placed at a given distance from the halogen $\mathrm{X}\left(\mathrm{d}_{\mathrm{X}} \cdots \mathrm{EP}\right)$ whereas the halogen $\mathrm{PB}$ radius, along with those of the remainder atoms, is used to define the region of the solute $\left(\epsilon_{\text {in }}\right)$ and the solvent $\left(\epsilon_{\text {out }}\right)$.

in drug discovery, ${ }^{20,23}$ mainly due to their propensity to improve drug-like properties such as membrane permeability and pharmacokinetic stability. ${ }^{24,25}$ Therefore, computational methods aiming at describing halogenated ligands and their association with a biomolecular target must provide a proper description of the $\sigma$-hole which is not straightforwardly achieved by standard force fields (FFs) as these typically consider halogen atoms to carry a negative charge leading to unfavorable interactions with other negative atoms. ${ }^{21}$ To overcome this problem, various strategies to emulate the $\sigma$-hole in empirical FFs emerged, namely, electric multipole expansions, aspherical interatomic potentials, and off-center point charges. ${ }^{26}$ The latter methodology is quite simple and consists of placing a positive charge, often called extra point (EP), at a given distance from the halogen along the $\mathrm{R}-\mathrm{X}$ bond axis to emulate the $\sigma$-hole (Figure 1). This strategy has the advantage of a rather low impact on the computational cost which is important for large-scale simulations. Thus, a variety of different implementations of the EP strategy were published ${ }^{27-34}$ differing in the charge-fitting scheme or charges assigned, and on the $\mathrm{X} \cdots \mathrm{EP}$ distance $\left(\mathrm{d}_{\mathrm{X}} \ldots \mathrm{EP}\right)$, being able to properly describe 
XBs in molecular dynamics simulations of protein-ligand systems. ${ }^{21}$ In some studies, MD trajectories were used in MM-(GB/PB)SA (GB = Generalized Born) calculations to estimate $\Delta G_{\text {bind }}{ }^{27,35-38}$ and even though the presence of an EP usually leads to accurate results regarding protein-ligand geometries and sampling of XBs, its impact on the accuracy of $\Delta G_{\text {solv }}$ predictions when using such methods is yet to be properly evaluated.

Very recently, ${ }^{39}$ we showed that a problematic issue arises when using PBSA calculations combined with a common off-center point-charge implementation for the General Amber Force Field $(\mathrm{GAFF})^{40}$ that uses a $\mathrm{d}_{\mathrm{X}} \ldots \mathrm{EP}$ value corresponding to the Lennard-Jones (LJ) parameter $R_{\min }$ of the halogen ${ }^{27}$ along with restrained electrostatic potential (RESP) charges $^{41}$ fitted for all particles (here denoted $\boldsymbol{E P} \mathbf{1}$ method). Indeed, since PB radii sets such as $\mathrm{PARSE}^{42}$ or modified Bondi (mbondi) ${ }^{43}$ do not include specific values for the heavier halogens, standard radii $\left(r_{\text {std }}\right)$ are commonly taken from the literature ${ }^{4,44}$ (Table S1). However, these values are smaller than $R_{\min }$, thus placing the EP outside the halogen radius, i.e., in the solvent dielectric, leading to a poor estimation of $\Delta G_{\text {solv }}$. By conducting an optimization we provided a new set of halogen PB radii $\left(r_{\mathrm{opt}}\right)$ that can be used with $\mathrm{d}_{\mathrm{X}} \cdots \mathrm{EP}=R_{\min }$ in the context of GAFF ( $E P 1)^{27}$ yielding mean absolute error (MAE) values between experimental and calculated $\Delta G_{\text {solv }}$ values lower than $1.5 \mathrm{kcal} \mathrm{mol}^{-1}$ as opposed to $4.2 \mathrm{kcal} \mathrm{mol}^{-1}$ when standard radii are used. This work also showed that even without EP addition, the halogen PB radii can be slightly optimized (MAE below $1.8 \mathrm{kcal} \mathrm{mol}^{-1}$ vs $2.0 \mathrm{kcal} \mathrm{mol}^{-1}$ without optimization). A grid of $\mathrm{d}_{\mathrm{X}} \ldots \mathrm{EP}$ values and halogen $\mathrm{PB}$ radii eventually showed the nonexistence of optimal parameters as a wide range of pairs yield low MAE values, also demonstrating that the optimization strategy can be ported into other FFs and/or off-center point-charge implementations.

As mentioned above, and apart from $\boldsymbol{E P 1}$, other EP strategies are available in the literature when a proper description of halogen bonding is needed at the MM/MD level. However, subsequent analysis of such simulations with (MM)-PBSA calculations is hindered due to the lack of specific PBSA parameters for these models. Therefore, in this paper, we assess the 
performance of PBSA in the estimation of $\Delta G_{\text {solv }}$ in water $\left(\Delta G_{\text {hyd }}\right)$ using three different offcenter point-charge implementations taken from the literature in the context of GAFF ${ }^{28,30}$ and $\mathrm{CHARMM}^{31}$ force fields, also conducting an optimization of the $\mathrm{PB}$ radii for $\mathrm{Cl}, \mathrm{Br}$, and I for each EP model. With this work, we contribute to improving the description of halogenated compounds in structure-based virtual screening approaches by providing methods that accurately estimate $\Delta G_{\text {solv }}$ values which are paramount to understand protein-ligand binding events. Moreover, and since the addition of EPs is not limited to halogens, being also important to address anisotropic electronic features in other elements such sulfur $(\sigma-$ hole), ${ }^{45,46}$ oxygen or nitrogen atoms (lone pairs), ${ }^{45,47}$ this work might also lay the ground for expanding the optimizations to those elements.

\section{Methods}

\section{Experimental Data}

From FreeSolv ${ }^{48}$ (version $0.51^{49}$ ), a database of experimental and simulation data of hydration free energies, 142 halogenated molecules were taken, comprising 107 chlorinated, 23 brominated, and 12 iodinated compounds which were analysed independently. As before, ${ }^{39}$ compounds containing multiple halogens from different elements in their structure (other than fluorine), namely, ID 4506634 (2-bromo-2-chloro-1,1,1-trifluoroethane) and ID 7768165 (1-bromo-2-chloroethane) were excluded from our analysis. Regarding fluorine-containing molecules, only compounds comprising also heavier halogens in their structure were considered.

\section{Off-center point-charge models}

We used three different off-center point-charge implementations taken from the literature termed $\boldsymbol{E P 2},{ }^{28} \boldsymbol{E P} 3,{ }^{30}$ and $\boldsymbol{E P 4} \cdot{ }^{31}$ For all of them, only one EP is added along the C$\mathrm{X}$ covalent bond axis with the $\mathrm{C}-\mathrm{X} \cdots \mathrm{EP}$ angle being fixed at $180.0^{\circ}$. Their main features, 
namely, $\mathrm{d}_{\mathrm{X}} \ldots \mathrm{EP}$ and atomic charges assigned to the EP ( $\left.\mathrm{qEP}_{\mathrm{EP}}\right)$ are summarized in Table 1 . The values for model $\boldsymbol{E P 1}$, studied in an earlier publication, ${ }^{39}$ are also shown for comparison. Table 1: General information ( $\mathrm{d}_{\mathrm{X}} \ldots \mathrm{EP}$ in $\AA$ and EP charges, $\left.\mathrm{q}_{\mathrm{EP}}\right)$ for the different EP models used in this work.

\begin{tabular}{|c|c|c|c|c|c|c|c|c|}
\hline & \multicolumn{4}{|c|}{ GAFF } & \multicolumn{4}{|c|}{ CHARMM } \\
\hline & \multicolumn{2}{|r|}{$E P 1$} & \multicolumn{2}{|c|}{$E P 2$} & \multicolumn{2}{|c|}{ EP3 } & \multicolumn{2}{|c|}{$\mathrm{EP}_{4}$} \\
\hline Halogen & $d_{X} \cdots E P$ & $\mathrm{q}_{\mathrm{EP}}$ & $d_{X} \cdots E P$ & $\mathrm{q}_{\mathrm{EP}}$ & $d_{X} \cdots E P$ & qEP & $d_{X} \cdots E P$ & qEP \\
\hline $\mathrm{Cl}$ & 1.948 & & & & 1.00 & 0.1 & 1.64 & 0.05 \\
\hline $\mathrm{Br}$ & 2.020 & RESP fitted & Best fit & $2 \mathrm{M} \mathrm{ESP}{ }^{a}$ & 1.30 & 0.2 & 1.89 & 0.05 \\
\hline I & 2.150 & & & & 1.60 & 0.3 & 2.20 & 0.05 \\
\hline
\end{tabular}

a The list of $\mathrm{dx}_{\mathrm{X}} \cdots \mathrm{EP}$ values is given in Tables $\mathrm{S} 2-\mathrm{S} 4$.

The first off-center point-charge model studied in this work was proposed by Sironi and co-workers $^{28}$ (Table 1, EP2) in the context of AMBER/GAFF. ${ }^{40}$ In this approach, atomic charges are fitted for all atoms (including the EP) using a RESP fitting procedure, and $\mathrm{d}_{\mathrm{X}} \cdots \mathrm{EP}$ values are determined individually for each molecule by minimizing the error of the fit to the reference quantum-mechanical (QM) electrostatic potential (ESP). Thus, using previously optimized geometries at the B3LYP/6-311G(d,p) level of theory ${ }^{50-55}$ with Gaussian 09, Revision A.2, ${ }^{56}$ the reference ESPs were generated at the HF/6-31G(d) ${ }^{57-59}$ level of theory for all elements, with the exception of iodine, for which the $6-311 \mathrm{G}(\mathrm{d})^{55}$ basis set, downloaded from the Basis Set Exchange website, ${ }^{60}$ was used. The remainder parameters are described in an earlier publication. ${ }^{39}$ Atomic partial charges were obtained by applying the restrained ESP (RESP) ${ }^{41}$ procedure using the antechamber ${ }^{61}$ module as implemented in AmberTools17. ${ }^{62}$ During this RESP fitting procedure, an EP was placed along the $\mathrm{C}-\mathrm{X}$ covalent bond by varying $\mathrm{d}_{\mathrm{X}} \ldots \mathrm{EP}$ from $1 \AA$ to $4 \AA$ with $0.01 \AA$ increments. According to the $\boldsymbol{E P} \mathcal{2}$ model strategy, the selected $\mathrm{d}_{\mathrm{X}} \ldots \mathrm{EP}$ values were the ones minimizing the relative root-mean-square (RRMS) error of the fit. When $\mathrm{d}_{\mathrm{X}} \ldots$ EP was larger than $R_{\min }$, the value of $R_{\min }$ and respective atomic charges were attributed instead. The final $\mathrm{d}_{\mathrm{X}} \ldots \mathrm{EP}$ values obtained for each compound are presented in Tables S2-S4. Notice that in $\boldsymbol{E P} \mathscr{2}$, and owing 
to the variable nature of the $\mathrm{d}_{\mathrm{X}} \cdots \mathrm{EP}$ values, the EP is often placed in the water dielectric when using standard halogen PB radii (Table S1), i.e. $\mathrm{d}_{\mathrm{X}} \cdots \mathrm{EP} P r_{\text {std }}$ (see also the Off-center point-charge model $\boldsymbol{E P} 2$ section in Results and Discussion).

The second method tested, EP3, also called "no fit" explicit $\sigma$-hole (ESH), was proposed by Hobza and co-workers ${ }^{30}$ in the context of AMBER/GAFF. ${ }^{40}$ In this work a Br-EP distance of $1.5 \AA$ and an EP charge of $0.20 e$ were suggested as universal parameters. These values were reviewed in a subsequent publication, ${ }^{63}$ also providing $\mathrm{d}_{\mathrm{X}} \cdots \mathrm{EP}$ and $\mathrm{qEP}_{\mathrm{EP}}$ values for iodine and chlorine. In this case, the same RESP fitting procedure as described before was performed without EP addition. Then, the EP was added at the corresponding $d_{X} \cdots E P$ value and a charge ( $\left.q_{E P}\right)$ was attributed (Table 1, EP3). The value of $q_{E P}$ is then subtracted from the halogen charge, while the other atomic partial charges were not modified. Notice that once charges are obtained for a given set of molecules, no additional calculations are necessary to assign EP charges, thus saving computational time which could be useful for high-throughput calculations. ${ }^{63}$ In this EP model, the $\mathrm{d}_{\mathrm{X}} \cdots$ EP values are always smaller than the corresponding $r_{\text {std }}$ and therefore, the EP is correctly placed within the solute dielectric.

The last method explored in this work, named $\boldsymbol{E P} 4$, is based on the parameterization of XBs in the CHARMM General Force Field (CGenFF). ${ }^{31}$ In this case, and similarly to $\boldsymbol{E P} 3$, the EP distances and charges for each halogen are fixed (Table 1, EP4). To the best of our knowledge, no specific halogen $\mathrm{PB}$ radii exist to be employed along with CGenFF parameters. In this work, we used the halogen $R_{\min }$ values as $r_{\text {std }}$ placeholders for the subsequent optimization (see Table S5 and Discussion below). Hence, using the standard

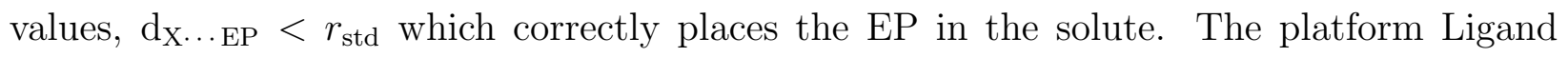
Reader \& Modeler ${ }^{64}$ from CHARMM-GUI ${ }^{65}$ or in some specific cases for which CHARMMGUI failed to create a topology file, the CGenFF server (https://cgenff.umaryland.edu) ${ }^{66}$ were used to generate CHARMM topologies containing atomic charges. Unfortunately for compounds ID 2996632 (chloroform), ID 6359135 (carbon-tetrachloride) and ID 8311321 (chloro-difluoro-methane), CHARMM topolgies could not be obtained, thus decreasing the 
number of chlorinated molecules analyzed to 104. A direct conversion of the CHARMM topology to an AMBER compatible one (e.g. using CHAMBER ${ }^{67}$ ), which could be read by the pbsa program of AmberTools 17, ${ }^{62}$ was not performed since colinear lonepairs are not supported. Therefore the CHARMM topologies were converted to a free-format pqr file using in-house scripts.

\section{PBSA Calculations}

We followed a similar procedure as the one described in reference 39. Succinctly, PBSA calculations were performed using the pbsa program provided in AmberTools $17,{ }^{62}$ with a single conformation per compound. These type of data sets are most frequently constituted by compounds with few rotatable bonds ${ }^{4}$ thus averaging over multiple conformations has only a marginal impact on the results. ${ }^{68}$ Indeed, in our previous work, ${ }^{39}$ no significant improvement is achieved after systematic conformational search for selected compounds. The solvation free energy $\left(\Delta G_{\text {solv }}\right)$ was computed from the contribution of a polar component $\left(\Delta G_{\text {polar }}\right)$ and a nonpolar component $\left(\Delta G_{\text {nonpolar }}\right)$ :

$$
\Delta G_{\text {solv }}=\Delta G_{\text {polar }}+\Delta G_{\text {nonpolar }}
$$

The $\Delta G_{\text {polar }}$ accounts for the electrostatic contribution to the total solvation free energy, obtained by a finite difference solution of the linearized Poisson-Boltzmann (PB) equation. On the other hand, the $\Delta G_{\text {nonpolar }}$ returns the contribution of solute-solvent hydrophobic interactions and cavitation. A solvent probe radius of $1.4 \AA$ was used to define the solvent excluded surface and the solute and solvent dielectric constants were set to 1 and 80 , respectively, thus setting $\Delta G_{\text {solv }}=\Delta G_{\text {hyd }}$. A default grid spacing of $0.5 \AA$ was used, which is common in simulations of large biomolecular systems where the optimized radii will be used. We verified that this value was not problematic for our calculations in small molecules with an EP. Indeed, the values calculated with the default grid $(0.5 \AA)$ do not differ substantially 
from those obtained using finer grids $(0.2 \AA$ and $0.1 \AA)$ for a few selected molecules using the different calculations setups (Table S6). The remainder PBSA parameters can be found in reference 39 .

In the context of AMBER/GAFF (methods $\boldsymbol{E P} 2, \boldsymbol{E P}$ 3) we used three different calculation setups, termed $\boldsymbol{p} \boldsymbol{b} \mathbf{1}, \boldsymbol{p} \boldsymbol{b} \boldsymbol{2}$, and $\boldsymbol{p} \boldsymbol{b} \mathbf{3}$, representing different flavors usually employed for MM-PBSA calculations with this force field (Table S7). Briefly, for $\boldsymbol{p} \boldsymbol{b} \boldsymbol{1}$ and $\boldsymbol{p} \boldsymbol{b} \boldsymbol{2}$ setups, the PARSE ${ }^{42}$ and mbondi radii, ${ }^{43}$ respectively, are used for both $\Delta G_{\text {polar }}$ and $\Delta G_{\text {nonpolar }}$ calculations. For $\boldsymbol{p} \boldsymbol{b} 3, \Delta G_{\text {polar }}$ is calculated using mbondi radii whereas for $\Delta G_{\text {nonpolar }}$, attractive $\left(\Delta G_{\text {dispersion }}\right)$ and repulsive interactions are treated separately. ${ }^{10,69}$ This is done by using a surface-based integration method for the $\Delta G_{\text {dispersion }}$ term while the molecular volume enclosed by SASA (SAV) is estimated for the calculation of the cavity repulsive term using the $R_{\min }$ values as atomic radii. Atomic radii were assigned by default by leap in AmberTools $17^{62}$ for each set (PARSE and mbondi), with minor corrections and the values are provided in Table S1.

In the context of CHARMM/CGenFF, we used a calculation setup, termed $\boldsymbol{p} \boldsymbol{b} 4$ (Table S7), similar to the one described in reference 70 which applied CHARMM36 (C36) and CGenFF to reproduce the experimental solvation free energies of a set of 70 molecules yielding an RMS of $2.5 \mathrm{kcal} \mathrm{mol}^{-1}$. Similarly to that work, herein both $\Delta G_{\text {polar }}$ and $\Delta G_{\text {nonpolar }}$ were calculated using the set of radii from Nina et al. ${ }^{71}$ and Banavali et al. ${ }^{72}$ (see Table S5) and setting $\gamma=0.005 \mathrm{kcal} \mathrm{mol}^{-1} \AA^{-2}$ while the constant term, $\beta$ was set to zero.

For all tested off-center point-charge models and PBSA setups, the PB radii of the halogen atoms $(\mathrm{Cl}, \mathrm{Br}, \mathrm{I})$ were varied between 1.00 and $4.00 \AA$ at incremental values of $0.01 \AA$ while keeping those of the remaining elements constant. A value of $0.00 \AA$ was assigned to both the EP PB radius and its $R_{\min }$ value (a detailed explanation on how to run PBSA calculations with an EP was given in an earlier publication). ${ }^{39}$ It is important to mention that in setups $\boldsymbol{p} \boldsymbol{b} \mathbf{1}, \boldsymbol{p} \boldsymbol{b} \boldsymbol{2}$, and $\boldsymbol{p} \boldsymbol{b} \boldsymbol{4}$ the variation of the halogen $\mathrm{PB}$ radius affects both $\Delta G_{\text {polar }}$ and $\Delta G_{\text {nonpolar }}$ terms. For $\boldsymbol{p} \boldsymbol{b} 3$, the halogen radius variation only affects $\Delta G_{\text {polar }}$, 
while the contribution of $\Delta G_{\text {nonpolar }}$ is constant as it only depends on the $R_{\text {min }}$ value.

The mean absolute error (MAE), calculated as

$$
\text { MAE }=n^{-1} \sum_{i=1}^{n} \mid \Delta G_{\text {solv }}(\text { calc })_{i}-\Delta G_{\text {solv }}(\exp )_{i} \mid
$$

was used to evaluate the accuracy of the solvation free energy values. It consists of the unsigned difference between the computed $\left(\Delta G_{\text {solv }}(\right.$ calc $\left.)\right)$ and experimental $\left(\Delta G_{\text {solv }}(\exp )\right)$ values, averaged for each entire subset of compounds (n).

\section{MD simulations}

To evaluate the effect of solute flexibility, molecular dynamics simulations (MD) were performed on selected molecules using the GROMACS software package, version 2020.4 (GPU implementation). ${ }^{73}$ The solutes were solvated with TIP3P ${ }^{74}$ water molecules in a rhombic dodecahedron box using three-dimensional periodic boundary conditions and the minimum image convention. Electrostatic interactions were treated with particle mesh Ewald $(\mathrm{PME})^{75}$ where the contributions in the real space were added up to $1.0 \mathrm{~nm}$ and using a Fourier grid spacing of $0.12 \mathrm{~nm}$. The van der Waals forces were truncated at $1.0 \mathrm{~nm}$ and all bonds were constrained using the P-LINCS algorithm. ${ }^{76}$ Energy minimization was performed in two steps using the steepest descent algorithm, the first step without constraints, the second with all bonds constrained. The initiation protocol consisted of 100 ps in a NVT ensemble with the temperature kept constant at $298.15 \mathrm{~K}$ using the Berendsen thermostat. ${ }^{77}$ MD production runs of 100 ns were performed using a NPT ensemble where the temperature was kept constant at $298.15 \mathrm{~K}$ using the v-rescale ${ }^{78}$ thermostat with a coupling constant of 0.10 ps while pressure was maintained at 1 bar using the Parrinello-Rahman barostat ${ }^{79}$ with a coupling constant of 2.0 ps. The first 20 ns of the NPT run were discarded as equilibration time and afterwards, 800 equally-spaced conformations were extracted from the MD trajectory. 


\section{Results and Discussion}

The solvation free energies $\left(\Delta G_{\text {solv }}\right)$ for a set of halogenated molecules taken from the FreeSolv database ${ }^{48}$ were estimated using PBSA calculations with different calculation setups $(\boldsymbol{p b} \mathbf{1}, \boldsymbol{p b} \mathscr{2}, \boldsymbol{p b} 3$, and $\boldsymbol{p b} 4)$. Three off-center point-charge models (EP2, EP3, and $\boldsymbol{E P} 4)$, meant to tackle the anisotropy of the halogens, were evaluated in the context of PBSA calculations aiming at the optimization of the halogen radii by comparing the calculated $\Delta G_{\text {solv }}$ values with the experimental ones. A comparison with the values obtained in a previous work using $\boldsymbol{E P 1 ^ { 3 9 }}$ is also performed. A discussion for each EP model is presented in the following sections.

\section{Off-center point-charge model $E P \mathscr{2}$}

As mentioned previously, model $\boldsymbol{E P} \mathscr{\mathcal { Q }}$ is based on the work of Sironi and co-workers, ${ }^{28}$ and considers the ability of the EP to reproduce the full anisotropy of the potential around the halogen by using the X $\cdots$ EP distance that yield the best fit to the QM electrostatic potential. This means that, for each compound, a specific X $\cdots$ EP distance is assigned along with atomic charges which are attributed to all atoms (including the EP) by the RESP procedure. We therefore applied this method to our library of halogenated compounds and the $\mathrm{X} \cdots \mathrm{EP}$ distances $\left(\mathrm{d}_{\mathrm{X}} \cdots \mathrm{EP}\right)$ that provide the best fit of the ESP against the QM potential are summarized in Tables S2-S4. Notice that for the subsequent PBSA calculations, when the obtained $\mathrm{d}_{\mathrm{X}} \ldots \mathrm{EP}$ values are larger than $R_{\min }, \mathrm{d}_{\mathrm{X}} \ldots \mathrm{EP}$ is set to the $R_{\text {min }}$ value since placing the EP beyond this value might lead to instabilities during MD simulations. ${ }^{26}$ The distribution of $\mathrm{d}_{\mathrm{X}} \ldots \mathrm{EP}$ values for each dataset is shown in Figure 2. For iodinated molecules, the assigned distances are in the $1.25-2.41 \AA$ range with a maximum at $\approx 2.02 \AA$, slightly

smaller than the $R_{\text {min }}$ value for iodine $(2.15 \AA)$ and slightly larger than $r_{\text {std }}(1.98 \AA$ for both PARSE and mbondi sets, see Table S1) meaning that for several molecules, $\mathrm{d}_{\mathrm{X} \cdots \mathrm{EP}}>r_{\text {std }}$. For the brominated dataset, the distances that led to the best fit to the QM are in a range 


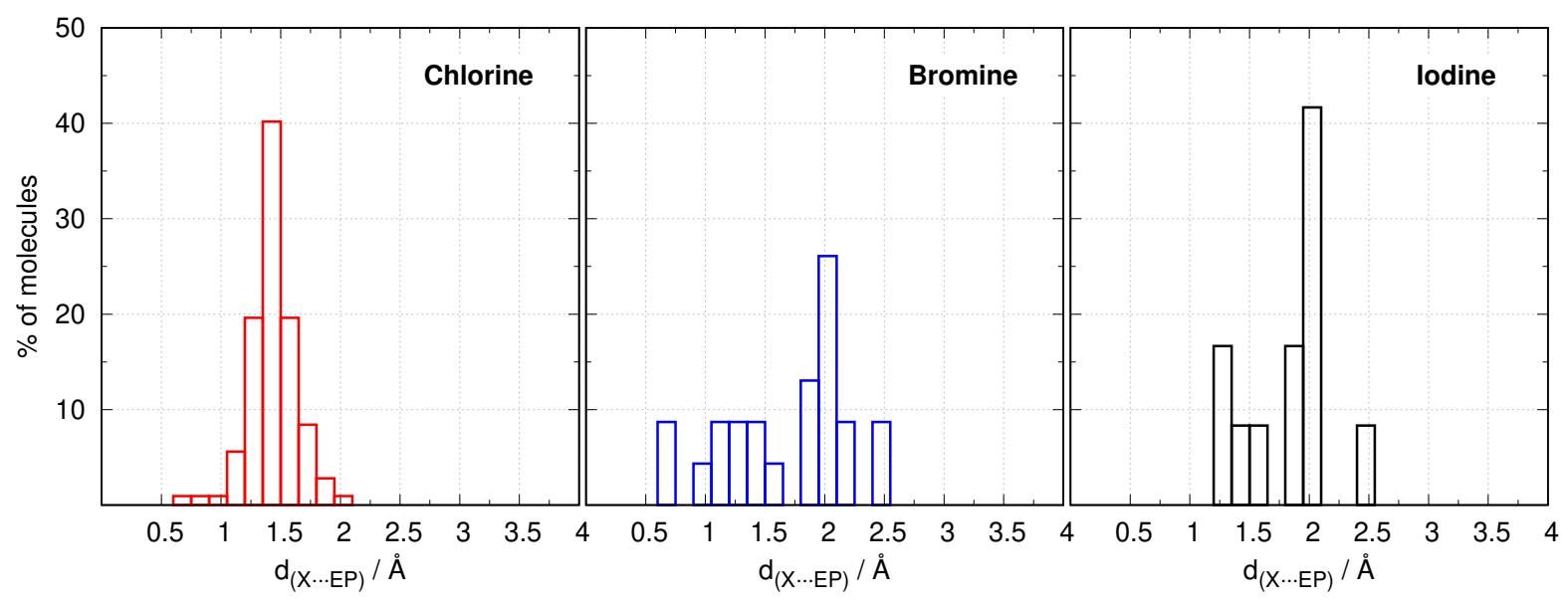

Figure 2: Distribution of $\mathrm{d}_{\mathrm{X}} \ldots \mathrm{EP}$ values $(\AA)$ assigned using $\boldsymbol{E P} \mathscr{2}$.

of $0.72-2.40 \AA$, with a peak occurring at $\approx 2.02 \AA$, similar to the $R_{\text {min }}$ value for bromine (2.02 $\AA$ ) and larger than the standard PB radius $(1.85 \AA)$. The behavior of the chlorinated set is different as the $\mathrm{d}_{\mathrm{X}} \ldots \mathrm{EP}$ values are evenly distributed around the maximum occurring at $1.42 \AA$ (range $0.74-1.97 \AA$ ), a value much smaller than the $R_{\min }$ for chlorine $(1.948 \AA$ ) and the standard PB radius $(1.70 \AA)$. These results show that placing the EP at $R_{\text {min }}$ in iodinated and brominated compounds (as in $\boldsymbol{E P 1}$ model) might not introduce larger errors on the fit of the ESP when compared with the reference potential, while for chlorinated molecules, shorter distances than $R_{\min }$ appear to reduce the mentioned error. Given the different size of the datasets, this issue must be further investigated, however, it is out of the scope of this manuscript.

In order to optimize the halogen $\mathrm{PB}$ radii, these were systematically varied for all compounds while using point charges derived for model $\boldsymbol{E P} \mathscr{2}$, and the MAE values against experimental values were calculated (Figure 3). The plot reflects the non-constant nature of the X ‥EP distances in model $\boldsymbol{E P} 2$. As we have shown before, ${ }^{39}$ when the halogen PB radius is equal to the $\mathrm{X} \cdots \mathrm{EP}$ distance, an asymptotic-like behavior in the error is observed. Therefore, several error peaks are observed whenever $\mathrm{PB}$ radii $=\mathrm{d}_{\mathrm{X}} \cdots \mathrm{EP}$ for a given compound. These errors may result from instabilities associated with a point charge being placed 


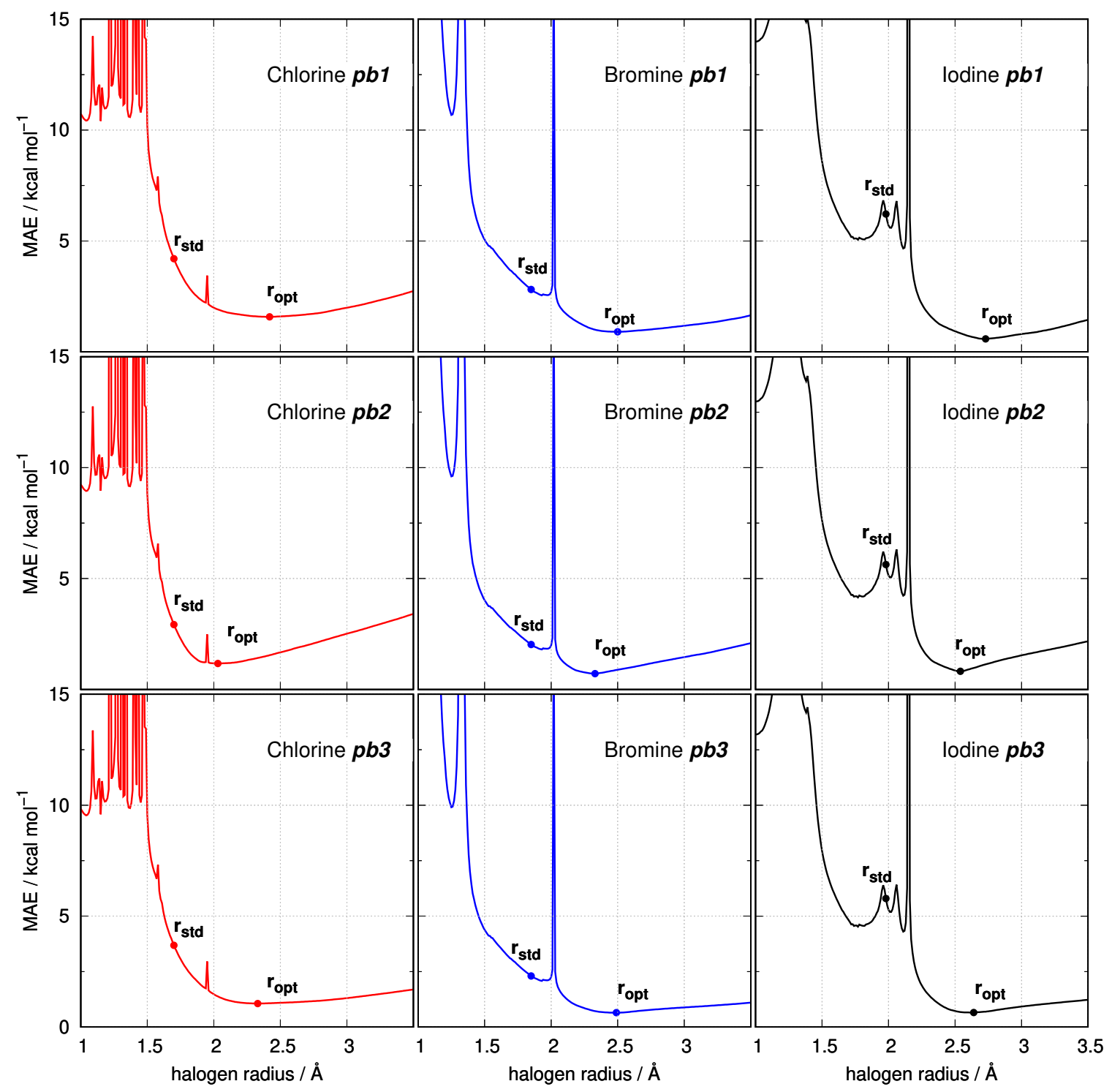

Figure 3: Variation of the MAE values with the halogen radius for each subset of halogenated compounds with an addition of an EP based in model $\boldsymbol{E P} 2$ using PBSA setups $\boldsymbol{p} \boldsymbol{b} \mathbf{1}, \boldsymbol{p} \boldsymbol{b} \boldsymbol{2}$, and $p \boldsymbol{b} 3$.

at the interface between the two-dielectric media. In spite the occurrence of these peaks, all curves show a minimum corresponding to an optimal halogen PB radius $\left(r_{\text {opt }}\right)$ that minimizes the MAE. The values are presented in Table 2 along with the MAEs obtained using $r_{\text {std }}$ values. Using standard radii leads to considerably larger errors (up to c.a. $6 \mathrm{kcal} \mathrm{mol}^{-1}$ 
Table 2: MAE $\left(\mathrm{kcal} \mathrm{mol}^{-1}\right)$ values obtained with standard $\left(r_{\mathrm{std}}\right)$ and optimized $\left(r_{\mathrm{opt}}\right)$ halogen radii $(\AA)$ for each subset of halogenated compounds with the addition of an EP using $\boldsymbol{E P} \mathscr{Z}$ model for PBSA setups $\boldsymbol{p} \boldsymbol{b} \mathbf{1}, \boldsymbol{p} \boldsymbol{b} \boldsymbol{2}$, and $\boldsymbol{p} \boldsymbol{b} 3$.

\begin{tabular}{|c|c|c|c|c|c|c|c|}
\hline \multirow{2}{*}{ Halogen } & \multirow{2}{*}{$r_{\text {std }}$} & \multicolumn{2}{|r|}{$p b 1$} & \multicolumn{2}{|r|}{$p b \mathscr{2}$} & \multicolumn{2}{|r|}{$p b 3$} \\
\hline & & $\mathrm{MAE}_{\mathrm{std}}$ & $\operatorname{MAE}_{\mathrm{opt}}\left(r_{\mathrm{opt}}\right)$ & $\mathrm{MAE}_{\mathrm{std}}$ & $\operatorname{MAE}_{\text {opt }}\left(r_{\text {opt }}\right)$ & $\mathrm{MAE}_{\mathrm{std}}$ & $\operatorname{MAE}_{\mathrm{opt}}\left(r_{\mathrm{opt}}\right)$ \\
\hline $\mathrm{Cl}$ & 1.70 & 4.201 & $1.579(2.42)$ & 2.923 & $1.172(2.03)$ & 3.686 & $1.059(2.33)$ \\
\hline $\mathrm{Br}$ & 1.85 & 2.812 & $0.901(2.50)$ & 2.025 & $0.712(2.33)$ & 2.302 & $0.647(2.49)$ \\
\hline I & 1.98 & 6.218 & $0.583(2.73)$ & 5.631 & $0.819(2.54)$ & 5.795 & $0.654(2.64)$ \\
\hline
\end{tabular}

for $\boldsymbol{p} \boldsymbol{b} \mathbf{1}$ in the iodine dataset) than those obtained using the optimized values for which the

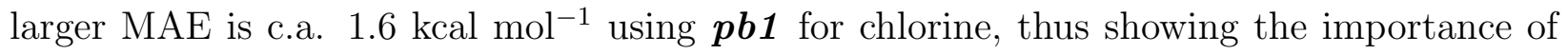
the halogen radii optimization in this context.

The setup that lead to lower MAE values for iodinated compounds was $\boldsymbol{p} \boldsymbol{b} \mathbf{1}$, with an MAE value of $0.583 \mathrm{kcal} \mathrm{mol}^{-1}$, although $\boldsymbol{p b} 3$ yields competitive results $\left(0.654 \mathrm{kcal} \mathrm{mol}^{-1}\right)$. This latter setup achieved better results $\left(0.647 \mathrm{kcal} \mathrm{mol}^{-1}\right.$ and $\left.1.059 \mathrm{kcal} \mathrm{mol}^{-1}\right)$ for brominated and chlorinated compounds, respectively. Moreover, for this latter dataset, larger MAEs were observed for all setups, which was also verified in the previous study, ${ }^{39}$ and is related to the larger sample size (107 compounds).

The $r_{\text {opt }}$ values follow the expected order of increasing atomic number $(\mathrm{Cl}<\mathrm{Br}<\mathrm{I})$ in all the setups and are larger than the corresponding $R_{\min }$, which is coherent with the results obtained in a previous work ${ }^{39}$ for $\boldsymbol{E P 1}$. Indeed, comparing the $\boldsymbol{E P} \mathscr{2}$ results with those reported for $\boldsymbol{E P 1}$, both the $r_{\text {opt }}$ values and MAEs are quite similar.

The correlation between the calculated and experimental $\Delta G_{\text {solv }}$ values can be obtained using $r_{\text {opt }}$ values (EP2, Figure 4). For all the setups, the correlations are high, although slightly lower for chlorinated compounds, which is consistent with the MAE values obtained. Additionally, the majority of the compounds possess an absolute deviation below $2 \mathrm{kcal} \mathrm{mol}^{-1}$ (Figure S1 and Figure S2), with higher deviations obtained for chlorinated compounds, as 


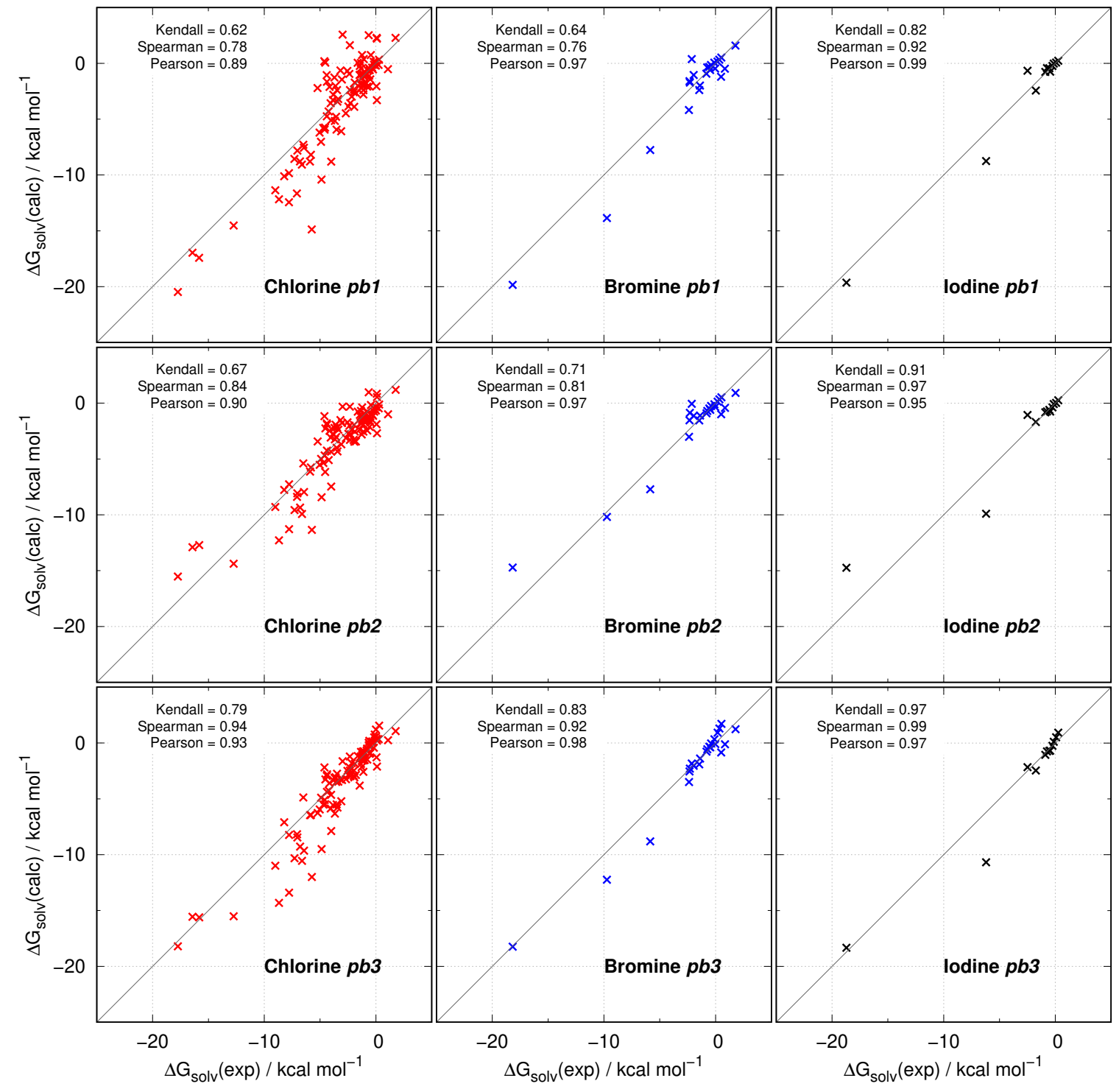

Figure 4: Correlation between experimental and calculated $\Delta G_{\text {solv }}$ values for each subset of halogenated compounds with with $\boldsymbol{E P} \mathscr{2}$ model. The inset shows the resulting Kendall $\tau$, Spearman rank correlation, and Pearson R correlation coefficients.

expected. These plots also show that the magnitude of the MAE is not concomitant with a systematic over/underestimation of $\Delta G_{\text {solv }}$ as the signed difference between calculated $\left(\Delta G_{\text {solv }}(\right.$ calc $\left.)\right)$ and the experimental values $\left(\Delta G_{\text {solv }}(\exp )\right)$ is reasonably distributed around 0. 
Although the ability to predict $\Delta G_{\text {solv }}$ significantly varies with the PBSA method, some compounds are particularly difficult to tackle, probably owing to the presence of elements and chemical groups whose effects are hard to tackle by continuum methods, e.g the formation of hydrogen bonds. For instance, in the iodinated set, 2-iodophenol presents the largest deviations ( -4.48 to $\left.-2.55 \mathrm{kcal} \mathrm{mol}^{-1}\right)$. This systematic overestimation also occurs in a brominated analogue, 4-bromophenol (deviations up to $-2.99 \mathrm{kcal} \mathrm{mol}^{-1}$ ). As before, ${ }^{39}$ the largest outlier is the chlorinated compound dialifor, the $\Delta G_{\text {solv }}$ values being highly overestimated especially when using the $\boldsymbol{p} \boldsymbol{b} \mathbf{1}$ setup $\left(-9.14 \mathrm{kcal} \mathrm{mol}^{-1}\right.$ deviation). Notice that the difficulties regarding the prediction of the hydration free energy of this compound was

observed in other studies, ${ }^{80,81}$ which can be associated with the presence of polar functional groups such as triophosphate, and also by a significant conformational floppiness. This latter hypothesis is not confirmed by ours results (see bellow).

Overall, using default radii leads to larger errors and therefore $r_{\text {opt }}$ values should be used instead. When comparing the three tested PBSA setups, $\boldsymbol{p b} 3$ setup is recommended under optimized conditions.

\section{Off-center point-charge model EP3}

Contrarily to $\boldsymbol{E P} 2$, in model $\boldsymbol{E P} 3$ the values for the distances and charges of the EP are fixed (Table 1). ${ }^{30}$ Thus, in the plots depicting the variation of the MAE against the PB radius of the halogen (Figure 5), error peaks are observed at $1.00 \AA, 1.30 \AA$, and $1.60 \AA$, for chlorinated, brominated, and iodinate compounds, respectively. As explained before, these peaks occur when the $\mathrm{PB}$ radius value is equal to the $\mathrm{X} \cdots \mathrm{EP}$ distance $\left(\mathrm{d}_{\mathrm{X}} \cdots \mathrm{EP}\right)$ and the overall shape of the curves resemble those obtained with $\boldsymbol{E P} 1$ where a fixed X $\cdots \mathrm{EP}$ distance (equal to $R_{\min }$ ) was also used, ${ }^{39}$ although the peaks obtained herein are broader, specially for iodine.

In Table 3, the MAE values obtained for both $r_{\text {std }}$ and $r_{\text {opt }}$ are summarized. For iodinated molecules, the errors obtained using $r_{\text {std }}$ values were significantly large (> c.a. $15 \mathrm{kcal} \mathrm{mol}^{-1}$ ) 


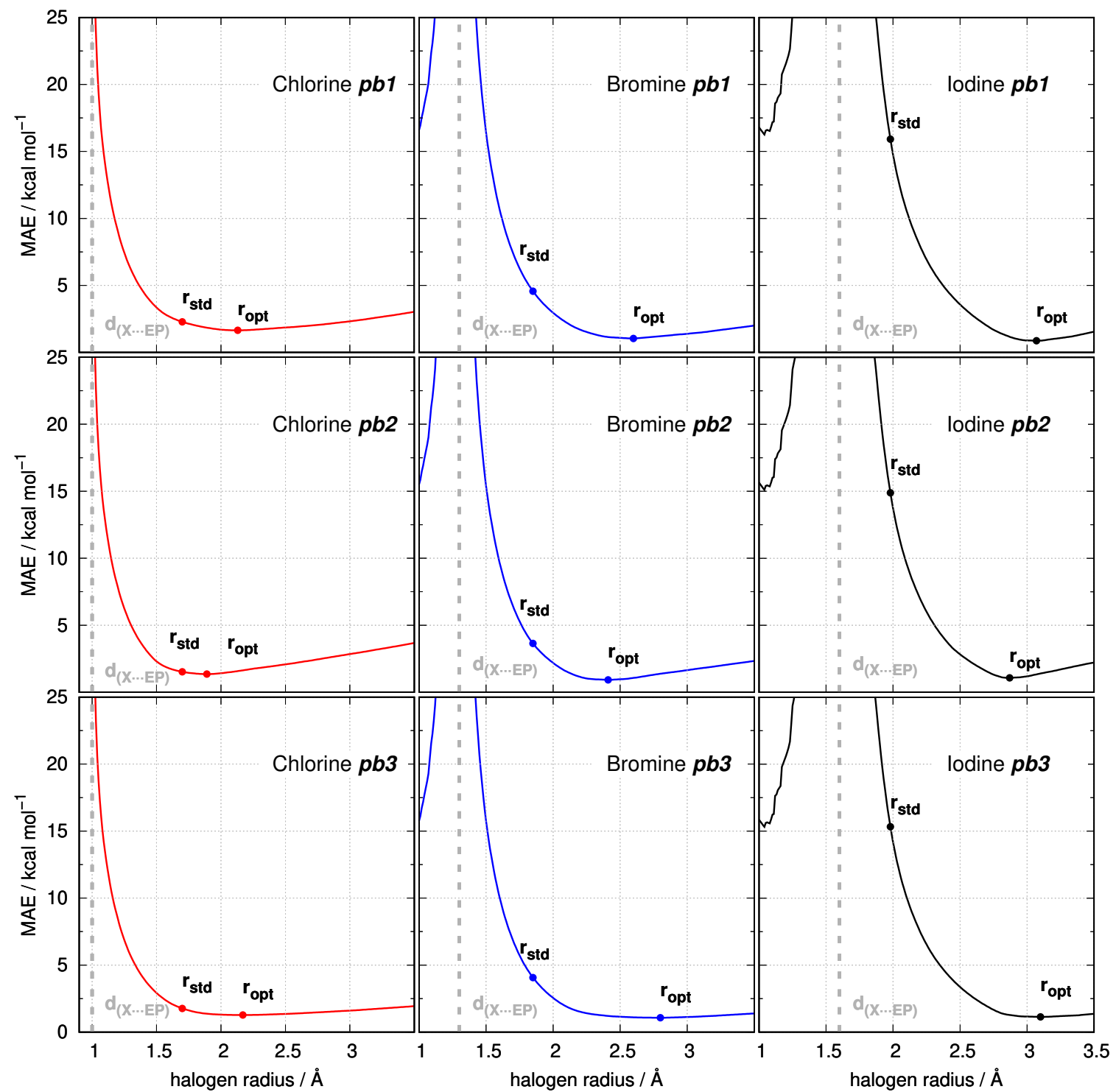

Figure 5: Variation of the MAE values with the halogen radius for each subset of halogenated compounds with an addition of an EP based in model $\boldsymbol{E P}$ 3 using PBSA setups $\boldsymbol{p} \boldsymbol{b} \boldsymbol{1}, \boldsymbol{p b} \boldsymbol{2}$, and $p b 3$. 
Table 3: MAE $\left(\mathrm{kcal} \mathrm{mol}^{-1}\right)$ values obtained with standard $\left(r_{\text {std }}\right)$ and optimized $\left(r_{\text {opt }}\right)$ halogen radii $(\AA)$ for each subset of halogenated compounds with the addition of an EP using $\boldsymbol{E P} 3$ model for PBSA setups $\boldsymbol{p} \boldsymbol{b} \mathbf{1}, \boldsymbol{p} \boldsymbol{b} \boldsymbol{2}$, and $\boldsymbol{p} \boldsymbol{b} 3$.

\begin{tabular}{|c|c|c|c|c|c|c|c|}
\hline \multirow{2}{*}{ Halogen } & \multirow{2}{*}{$r_{\text {std }}$} & \multicolumn{2}{|r|}{$p b 1$} & \multicolumn{2}{|r|}{$p b \mathcal{2}$} & \multicolumn{2}{|r|}{$p b 3$} \\
\hline & & $\mathrm{MAE}_{\mathrm{std}}$ & $\mathrm{MAE}_{\mathrm{opt}}\left(r_{\mathrm{opt}}\right)$ & $\mathrm{MAE}_{\mathrm{std}}$ & $\operatorname{MAE}_{\mathrm{opt}}\left(r_{\mathrm{opt}}\right)$ & $\mathrm{MAE}_{\mathrm{std}}$ & $\operatorname{MAE}_{\text {opt }}\left(r_{\text {opt }}\right)$ \\
\hline $\mathrm{Cl}$ & 1.70 & 2.281 & $1.648(2.13)$ & 1.525 & $1.352(1.89)$ & 1.766 & $1.273(2.17)$ \\
\hline $\mathrm{Br}$ & 1.85 & 4.564 & $1.034(2.60)$ & 3.639 & $0.924(2.41)$ & 4.067 & $1.069(2.80)$ \\
\hline I & 1.98 & 15.92 & 0.869 (3.07) & 14.89 & $1.074(2.87)$ & 15.33 & $1.131(3.10)$ \\
\hline
\end{tabular}

for all tested PBSA setups. This is caused by the fact that $r_{\text {std }}$ is located very near the broad error peak. Thus, the standard PB radii for iodine $(1.98 \AA)$ along with and a X $\cdots \mathrm{EP}$ distance of $1.6 \AA$ without charge refitting is not recommended in spite of the distances fulfill the criterion of placing the off-center point-charge inside the solute dielectric $\left(r_{\mathrm{opt}}>\mathrm{d}_{\mathrm{X}} \cdots \mathrm{EP}\right)$, again, highlighting the importance of the halogen PB radii optimization. Indeed, by using the optimized iodine $\mathrm{PB}$ radii, much lower MAE values (c.a. $1 \mathrm{kcal} \mathrm{mol}^{-1}$ ) are obtained. For brominated molecules, the MAE values obtained using standard PB radii are also relatively large (c.a. $4 \mathrm{kcal} \mathrm{mol}^{-1}$ ) whereas, noticeably, those obtained for the chlorinated database are not particularly high $\left(\approx 2 \mathrm{kcal} \mathrm{mol}^{-1}\right)$ since $r_{\text {std }}$ is substantially far from the broad error peak located a $1.00 \AA$ (Figure 5). Nonetheless, the MAE values decrease, specially in brominated molecules, when optimized halogen PB radii are used.

Again, for all the PBSA setups, the $r_{\text {opt }}$ values follow the expected order of increasing atomic number $(\mathrm{Cl}<\mathrm{Br}<\mathrm{I})$ and the setup that leads to lower MAE errors is dependent on the halogen set. For iodinated molecules, $\boldsymbol{p b} \mathbf{1}$ performs better $\left(\mathrm{MAE}=0.869 \mathrm{kcal} \mathrm{mol}^{-1}\right)$, while for brominated and chlorinated, $\boldsymbol{p} \boldsymbol{b} \mathscr{2}$ an $\boldsymbol{p} \boldsymbol{b} \boldsymbol{3}$ yield better results $\left(0.924 \mathrm{kcal} \mathrm{mol}^{-1}\right.$ and $1.273 \mathrm{kcal} \mathrm{mol}^{-1}$, respectively). These are, however, slightly higher than those obtained for $\boldsymbol{E P} \mathscr{2}$ in this work and for $\boldsymbol{E P} \mathbf{1}$ in an earlier study, ${ }^{39}$ although still acceptable, specially taking into account that in model EP3 the charges are not specifically fitted with the 
EP. Nonetheless, a recommendation of the PBSA flavour that should be used with this off-center point charge model is not evident. By plotting the calculated and experimental $\Delta G_{\text {solv }}$ values under optimized conditions (Figure 6), the correlations are high, which was expected considering the low MAE values. However, $\boldsymbol{p b} 3$ yields higher rank correlations for all halogens when compared with $\boldsymbol{p} \boldsymbol{b} \mathbf{1}$ and $\boldsymbol{p} \boldsymbol{b} \mathscr{2}$. It is also noteworthy to mention that our library of compounds qualitatively mimics the amount of each halogenated species present in the majority of databases, i.e. a huge presence of chlorinated molecules in relation to iodinated molecules. If we consider the weighted average of the MAEs for each setup $(\bar{x}=$ $1.489 \mathrm{kcal} \mathrm{mol}^{-1}$ for $\boldsymbol{p} \boldsymbol{b} \mathbf{1} ; \overline{\boldsymbol{x}}=1.264 \mathrm{kcal} \mathrm{mol}^{-1}$ for $\boldsymbol{p} \boldsymbol{b} \boldsymbol{2} ; \overline{\boldsymbol{x}}=1.230 \mathrm{kcal} \mathrm{mol}^{-1}$ for $\left.\boldsymbol{p} \boldsymbol{b} \mathbf{3}\right)$, and the mentioned correlations (Figure 6) the latter one performs the best. As for $\boldsymbol{E P} \mathscr{2}$, no obvious systematic over/underestimation of $\Delta G_{\text {solv }}$ is observed regardless of the used method (Figure S3 and Figure S4).

We also checked for systematic outliers on each setup/halogenated database. Again 2iodophenol was the compound that yielded higher deviations $\left(-5.60\right.$ to $\left.-3.58 \mathrm{kcal} \mathrm{mol}^{-1}\right)$, independently of the PBSA setup, while for brominated species, 4-bromophenol and bromacil are constantly ranked in the higher deviations (up to $-2.94 \mathrm{kcal} \mathrm{mol}^{-1}$ and $-5.13 \mathrm{kcal} \mathrm{mol}^{-1}$, respectively). Once more in the chlorinated dataset, the calculated $\Delta G_{\text {solv }}$ values for dialifor were considerably more negative than the experimental ones resulting in a high deviation (up to $-9.39 \mathrm{kcal} \mathrm{mol}^{-1}$ in $\boldsymbol{p b 1}$ ). Again, larger errors may arise from the presence of polar functional groups.

\section{Off-center point-charge model $E P 4$}

In order to test an off-center point-charge model out of the scope of AMBER/GAFF ${ }^{40}$ such as $\boldsymbol{E P} 2$ and $\boldsymbol{E P}$ 3, and given the similarity of the AMBER force field energy functional form with that of CHARMM, ${ }^{67}$ we also performed an optimization of the halogen $\mathrm{PB}$ radii in the context of this latter force field. Indeed, recently, the CHARMM General Force Field (CGenFF) was updated with positively-charged virtual particles to describe halogen bonds ${ }^{31}$ 


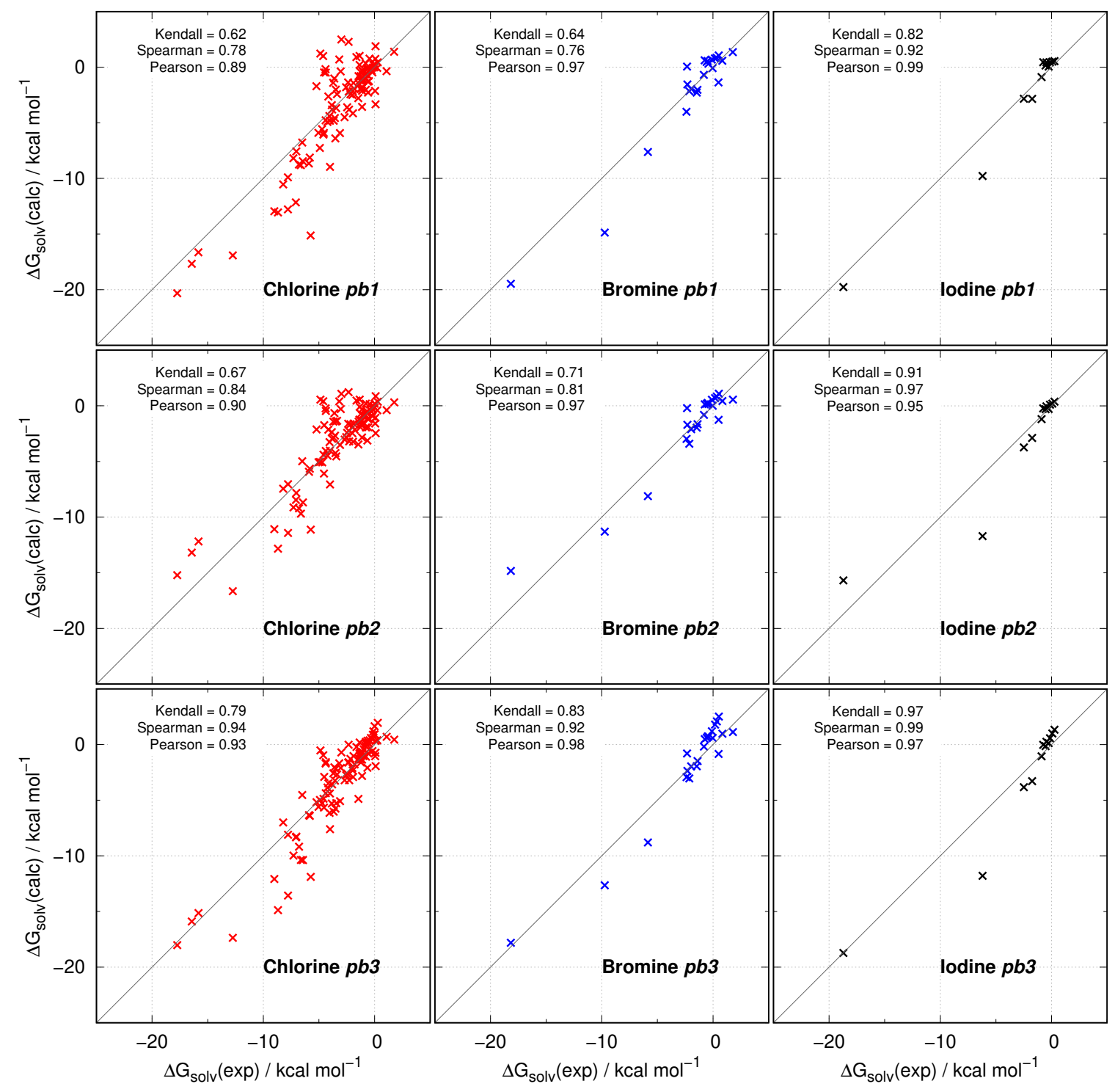

Figure 6: Correlation between experimental and calculated $\Delta G_{\text {solv }}$ values for each subset of halogenated compounds with with $\boldsymbol{E P} 3$ model. The inset shows the resulting Kendall $\tau$, Spearman rank correlation, and Pearson R correlation coefficients. 
with fixed charges $\left(\mathrm{q}_{\mathrm{EP}}\right)$ and distances $\left(\mathrm{d}_{\mathrm{X}} \ldots \mathrm{EP}\right)$ as summarized in Table 1. Although our set of halogenated molecules is diverse, it is important to notice that the reported EP parameterization was based on interactions in model systems of halobenzenes and therefore, the strategy of porting the parameters to other molecules should be subject of further analysis. The variations of the MAEs with halogen radius using PBSA setup $\boldsymbol{p} \boldsymbol{b} \boldsymbol{4}$ is depicted in Figure 7. Not surprisingly, the behavior of the curves is quite similar to the one observed for

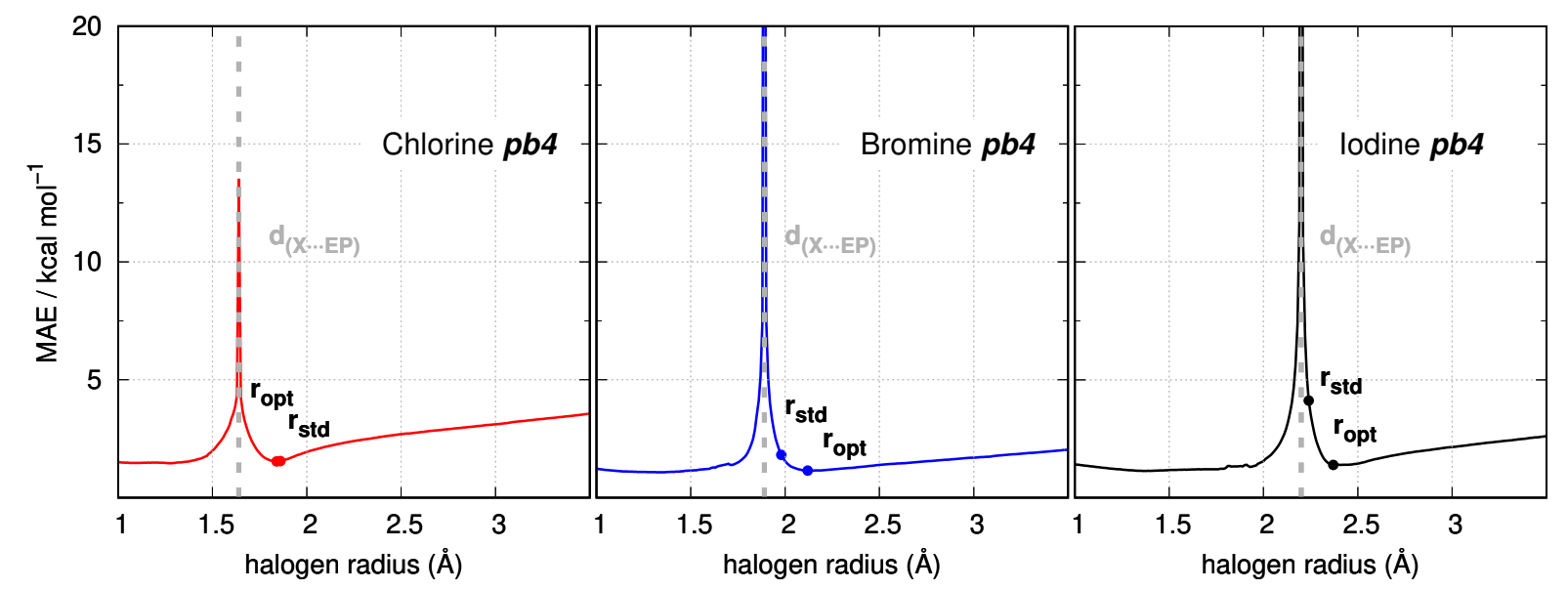

Figure 7: Variation of the MAE values with the halogen radius for each subset of halogenated compounds with an addition of an EP based in model EP 4 using PBSA setup $\boldsymbol{p b} 4$.

EP3 (this work) and $\boldsymbol{E P 1}$ (reference 39) where fixed $\mathrm{d}_{\mathrm{X}} \ldots \mathrm{EP}$ values are used. When the $\mathrm{PB}$ radius $=\mathrm{d}_{\mathrm{X} \cdots \mathrm{EP}}$, an error peak is observed while a minimum is observed at $\mathrm{PB}$ radius $>\mathrm{d}_{\mathrm{X}} \cdots \mathrm{EP}$. Table 4 reports the MAEs for obtained with $r_{\text {std }}$ along with those obtained with $r_{\text {opt }}$ while Table S8 presents values obtained without the addition of EP using a legacy version of $\mathrm{CGenFF}^{66}$ for comparison (some molecules were excluded ${ }^{82}$ ). Recall that while for the remainder elements CHARMM-compatible radii were used, ${ }^{71,72}$ for the halogens their $R_{\min }$ value was used as $r_{\text {std }}$. Surprisingly, for chlorine in the presence of the EP, the standard radii $(1.86 \AA)$ is extremely similar to the optimized one $(1.84 \AA)$, yielding MAEs of $\approx 1.5 \mathrm{kcal} \mathrm{mol}^{-1}$. For bromine with EP, radii optimization leads to a slight improvement of the MAE values whereas for iodine, a large improvement of about 3 orders of magnitude is 
Table 4: MAE $\left(\mathrm{kcal} \mathrm{mol}^{-1}\right)$ values obtained with standard $\left(r_{\text {std }}\right)$ and optimized $\left(r_{\text {opt }}\right)$ halogen radii $(\AA)$ for each subset of halogenated compounds with the addition of an EP using $\boldsymbol{E P} 4$ model for PBSA setup pb4

\begin{tabular}{cccc}
\hline \multirow{2}{*}{ Halogen } & $r_{\text {std }}$ & \multicolumn{2}{c}{$\boldsymbol{p} \boldsymbol{b} \boldsymbol{4}$} \\
\cline { 3 - 4 } & & $\mathrm{MAE}_{\text {std }}$ & $\mathrm{MAE}_{\text {opt }}\left(r_{\text {opt }}\right)$ \\
\hline $\mathrm{Cl}$ & 1.86 & 1.552 & $1.535(1.84)$ \\
$\mathrm{Br}$ & 1.98 & 1.816 & $1.148(2.12)$ \\
$\mathrm{I}$ & 2.24 & 4.120 & $1.392(2.37)$ \\
\hline
\end{tabular}

observed upon optimization owing to the fact that $r_{\text {std }}(2.24 \AA)$ is almost overlapping with $\mathrm{d}_{\mathrm{X} \cdots \mathrm{EP}}(2.20 \AA)$.

Comparing with $\boldsymbol{E P} 4$ (Table 4), the MAE values obtained without EP addition (Table S8) and using $r_{\text {std }}$ values are larger for all halogens, apart from iodine. The trend is observed under optimized conditions but in this case, comparable results are observed for iodine (1.392 kcal mol${ }^{-1}$ with $\boldsymbol{E P} 4,1.289 \mathrm{kcal} \mathrm{mol}^{-1}$ with no EP). Strikingly, the optimized radii without EP addition are too small $(<1.5 \AA)$ and do not follow the expected trend. Therefore, these results without EP addition will not be further considered for the subsequent analysis.

A strong linear correlation between calculated (using $\boldsymbol{E P} 4$ ) and experimental values (Figure 8) is obtained for iodine and bromine with Pearson correlation coefficients $>0.9$, tough a degradation of the monotonic character of the relationship is observed (lower Spearman rank correlation coefficients). For chlorine, the linear correlation (0.76) is the worse amongst tested PBSA setups and EP models and the same degradation of the monotonic character is observed.

Opposed to what was observed in the previous sections, using $\boldsymbol{E P} 4$ along with PBSA setup $\boldsymbol{p} \boldsymbol{b} \mathbf{4}$ leads to an underestimation of the calculated values when the experimental $\Delta G_{\text {solv }}$ values are highly negative (Figure S5). Moreover, while for chlorine and bromine the dif- 


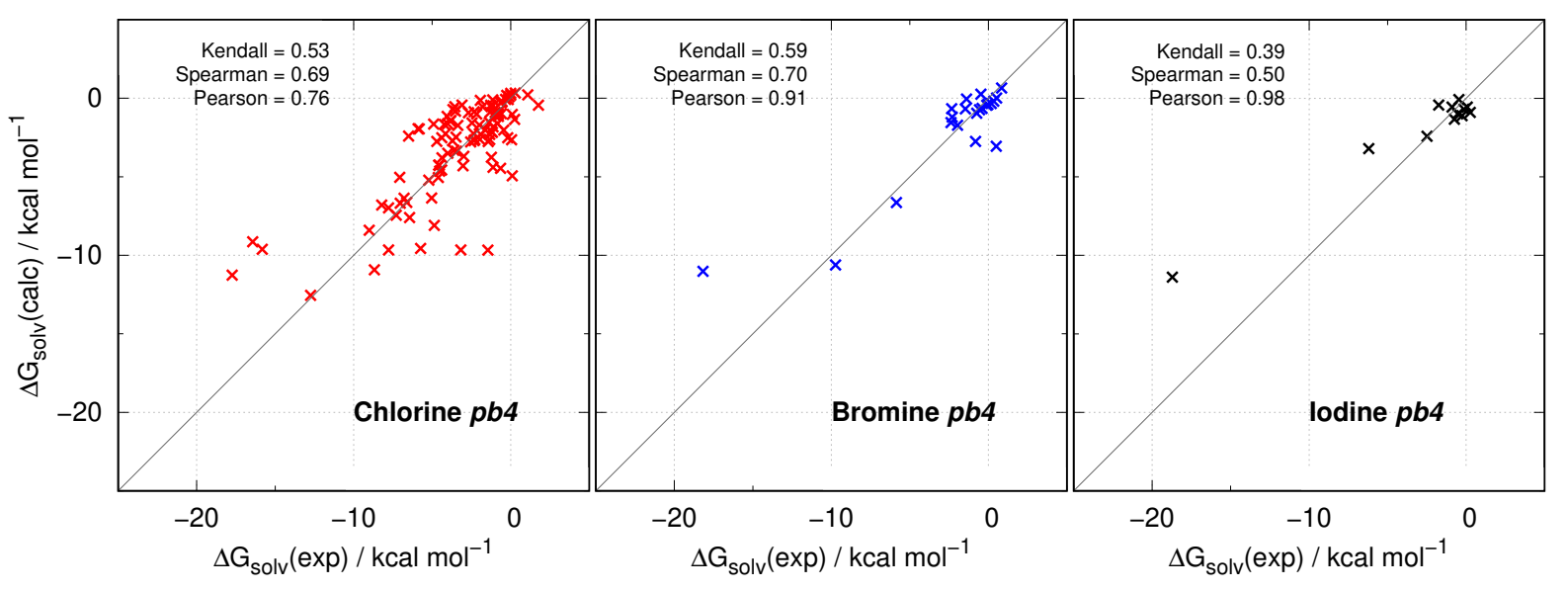

Figure 8: Correlation between experimental and calculated $\Delta G_{\text {solv }}$ values for each subset of halogenated compounds with with $\boldsymbol{E P} 4$ model and $\boldsymbol{p b} 4$ PBSA setup. The inset shows the resulting Kendall $\tau$, Spearman rank correlation, and Pearson R correlation coefficients.

ference between $\Delta G_{\text {solv }}\left(\right.$ calc) and $\Delta G_{\text {solv }}(\exp )$ values is reasonably distributed around 0 , for iodine the distribution is tilted towards positive values (Figure S6) owing to a large underestimation of $\Delta G_{\text {solv }}$ for 2-iodophenol (experimental $-6.20 \mathrm{kcal} \mathrm{mol}^{-1}$ vs calculated -3.19 $\mathrm{kcal} \mathrm{mol}^{-1}$ ) and 5-iodouracil (experimental -18.72 $\mathrm{kcal} \mathrm{mol}^{-1}$ vs $-11.39 \mathrm{kcal} \mathrm{mol}^{-1}$ ). For brominated compounds the largest deviation is observed for 5-bromouracil (an underestimation of c.a. $7 \mathrm{kcal} \mathrm{mol}^{-1}$ ) whereas 4-bromophenol and bromacil which were difficult cases using the previous EP models/PBSA setups are now nicely predicted (deviations of $-0.79 \mathrm{kcal} \mathrm{mol}^{-1}$ and $-0.89 \mathrm{kcal} \mathrm{mol}^{-1}$, respectively). For chlorinated molecules, the difficult case of dialifor has only a moderate deviation $\left(-3.82 \mathrm{kcal} \mathrm{mol}^{-1}\right)$ specially taking into account the deviations found in the other EP models while the largest outlier is now trichloronitromethane (experimental $-1.45 \mathrm{kcal} \mathrm{mol}^{-1}$, calculated $-9.67 \mathrm{kcal} \mathrm{mol}^{-1}$ ).

\section{Effect of the solute flexibility and $\Delta G_{\text {nonpolar }}$ contributions}

As mentioned earlier, we used a single conformation per compound which is a common strategy used in the literature for molecules with few rotatable bonds, ${ }^{83,84}$ specially when 
parameter optimization is being performed, ${ }^{4,39}$ thus reducing computational time and the number of degrees of freedom. Typically, averaging over multiple conformations in this type of datasets has a small impact on the results whereas Boltzmann sampling is expected to be relevant for large and more flexible molecules. ${ }^{68}$ Nonetheless, to assess the effect of solute flexibility, we selected 1-iodoheptane, 1-bromooctane, and dialifor, featuring 5, 6, and 8 rotatable bonds, respectively, and corresponding to the most flexible molecules of each dataset (Figure 9), one of them yielding the largest deviations against experimental values (dialifor). For each selected molecule, molecular dynamics simulations in explicit solvent

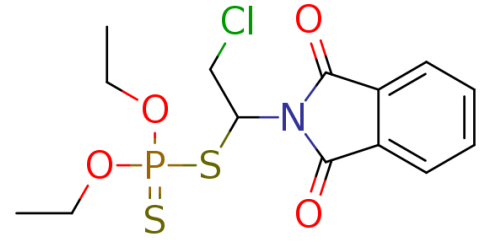

dialifor

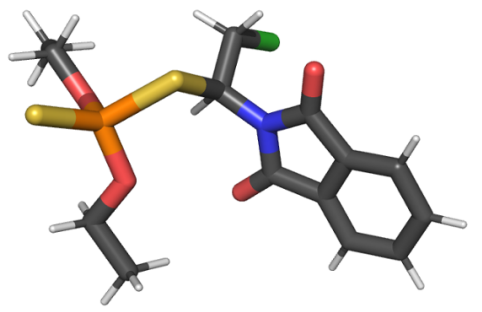

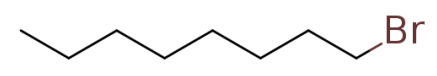

1-bromooctane

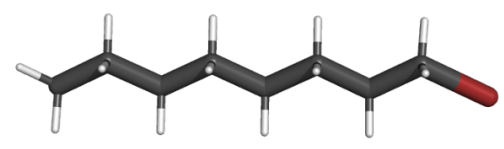

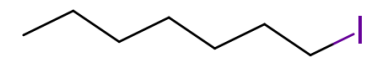

1-iodoheptane

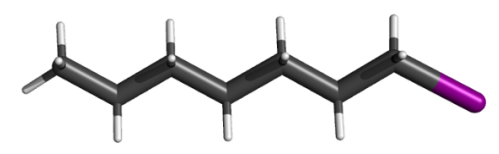

Figure 9: Selected molecules to evaluate the effect of solute flexibility: dialifor, 1bromooctane, and 1-iodoheptane.

were performed, using $\boldsymbol{E P} \mathscr{2}$ with optimized radii, extracting 800 equally-spaced conformations. For each conformation, $\Delta G_{\text {solv }}$ was then calculated using $\boldsymbol{p} \boldsymbol{b} \mathbf{1}$ - $\boldsymbol{p} \boldsymbol{b} \mathbf{3}$ and the results are summarized in Table 5, compared to those reported in Freesolv using MD simulations in explicit solvent and the Multistate Bennett Acceptance Ratio (MBAR) approach. ${ }^{85}$ The variation of $\Delta G_{\text {solv }}$ with the conformation along with the distribution of calculated values is shown in Figures S7 - S9.

For dialifor, which presents the largest number of rotatable bonds (8), the addition of 
Table 5: PBSA-calculated free energies of hydration $\left(\Delta G_{\text {solv }} / \mathrm{kcal} \mathrm{mol}^{-1}\right)$ using a single conformation $(\mathrm{n}=1)$ and an ensemble of structures (MD) obtained under optimized structures using $\boldsymbol{E P} \mathscr{2}$, compared with experimental values (exp \pm the experimental uncertainty, when available). The MD values correspond to the sample average $\pm 2 \sigma(\mathrm{n}=800, \sigma=$ sample standard deviation). Calculated values from Freesolv using MD simulations, explicit solvent and the Multistate Bennett Acceptance Ratio (MBAR) approach are also shown (FreeSolv/MBAR). ${ }^{85}$

\begin{tabular}{ccccccccc}
\hline & & & \multicolumn{2}{c}{$\boldsymbol{p} \boldsymbol{b} \mathbf{1}$} & \multicolumn{2}{c}{$\boldsymbol{p b} \boldsymbol{2}$} & \multicolumn{2}{c}{$\boldsymbol{p b} \boldsymbol{3}$} \\
\cline { 3 - 8 } Compound & $\exp$ & FreeSolv/Mbar & $\mathrm{n}=1$ & $\mathrm{MD}$ & $\mathrm{n}=1$ & $\mathrm{MD}$ & $\mathrm{n}=1$ & $\mathrm{MD}$ \\
\hline dialifor & $-5.75 \pm 1.93$ & $-16.52 \pm 0.07$ & -14.88 & $-16.06 \pm 2.62$ & -11.35 & $-12.45 \pm 2.46$ & -12.00 & $-12.82 \pm 2.64$ \\
1-bromooctane & 0.52 & $1.35 \pm 0.03$ & 0.52 & $0.29 \pm 0.30$ & 0.52 & $0.42 \pm 0.30$ & 1.71 & $0.78 \pm 0.38$ \\
1-iodoheptane & 0.27 & $0.23 \pm 0.03$ & 0.23 & $-0.06 \pm 0.54$ & 0.27 & $0.05 \pm 0.5$ & 0.94 & $0.18 \pm 0.54$ \\
\hline
\end{tabular}

conformational sampling did not improve nor changed the PBSA results when compared with those obtained using a single conformation, independently of the used setup. Indeed, the large deviation between the experimental value $\left(-5.74 \mathrm{kcal} \mathrm{mol}^{-1}\right)$ and PBSA-calculated values (ranging from $-11.35 \mathrm{kcal} \mathrm{mol}^{-1}$ to $-16.06 \mathrm{kcal} \mathrm{mol}^{-1}$ ) cannot be attributed to a lack of conformational sampling nor to the PBSA method since the calculated value reported in FreeSolv (-16.52 $\left.\mathrm{kcal} \mathrm{mol}^{-1}\right)$, obtained using explicit solvent simulations and the Multistate Bennett Acceptance Ratio (MBAR) approach ${ }^{85}$ is also similar and highly deviated. For 1-bromooctane, and using $\boldsymbol{p b} \mathbf{1}$, the deviation to the experimental value slightly increases when solute flexibility is taken into account, however, the MD values are still within the experimental and single conformation range as seen by the interval estimate (95\% confidence). The values obtained using $\boldsymbol{p} \boldsymbol{b} \mathscr{2}$ are practically invariant whereas using $\boldsymbol{p} \boldsymbol{b} 3$, adding conformational sampling clearly reduced the deviation to the experimental value, improving the calculated result. Curiously, $\Delta G_{\text {solv }}$ calculated with $\boldsymbol{p b} \mathbf{3}$ using a single conformation $\left(1.71 \mathrm{kcal} \mathrm{mol}^{-1}\right)$ is similar to the calculated one reported in FreeSolv $\left(1.35 \mathrm{kcal} \mathrm{mol}^{-1}\right)$. Concerning 1-iodoheptane, there is a slight increase in the deviation with respect to experimental values using $\boldsymbol{p b} \boldsymbol{1}$ and $\boldsymbol{p} \boldsymbol{b} \boldsymbol{2}$. Strikingly, and contrasting with all the previous cases, the $\Delta G_{\text {solv }}$ calculated values are not evenly distributed around the average value (Figure S9), the distribution appearing bimodal and the average value corresponding to a region of low probability. Nonetheless, and considering the confidence intervals, the MD values are still 
within both the experimental and single conformation values. In contrast, using $\boldsymbol{p} \boldsymbol{b} \mathbf{3}$, the calculated $\Delta G_{\text {solv }}$ are again normally distributed and the average value from the MD simulation improves the estimation of $\Delta G_{\text {solv }}$ when compared with the calculated value using a single conformation. Overall, the calculated $\Delta G_{\text {solv }}$ values using a single conformation are typically within the confidence intervals of the MD results. As mentioned above, the usage of a single conformation is common on such datasets and reduces the degrees of freedom in the optimization procedure. Nonetheless, and in spite of no systematic changes are observed for dialifor (all setups) and 1-bromooctane (in some setups), it is indeed true that small artifacts could arise from using a single conformation, specially in the case of long-chain linear haloalkanes, which deserves further exploring in future works.

The usage of different radii sets and methodologies to evaluate $\Delta G_{\text {nonpolar }}$ within the same force field, as performed with setups $\boldsymbol{p} \boldsymbol{b} \mathbf{1}-\boldsymbol{p} \boldsymbol{b} 3$, also offers an opportunity to evaluate the contribution of $\Delta G_{\text {nonpolar }}$ to the calculated $\Delta G_{\text {solv }}$ values. The variation of the calculated

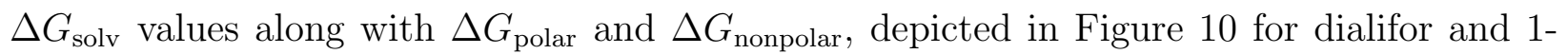
bromooctane using $\boldsymbol{E P} 2$ with optimized radii, shows that $\Delta G_{\text {nonpolar }}$ is basically invariant for the sampled conformations for setups $\boldsymbol{p} \boldsymbol{b} \mathbf{1}$ and $\boldsymbol{p} \boldsymbol{b} \boldsymbol{2}$. In these cases, the variation of $\Delta G_{\text {solv }}$ values is typically dictated by the $\Delta G_{\text {polar }}$ component. In contrast, when attractive $\left(\Delta G_{\text {dispersion }}\right)$ and repulsive interactions are taken into account $(\boldsymbol{p} \boldsymbol{b} 3)$, the $\Delta G_{\text {nonpolar values }}$ start to oscillate along the conformational space, this oscillation being more visible in the 1-bromooctane case.

As we noticed in our earlier study, ${ }^{39}$ often the negative (attractive) contribution of $\Delta G_{\text {dispersion }}$ yields a greater accuracy of the results. Since dialifor is a difficult case to handle, being the largest outlier for all methods/setups apart from $\boldsymbol{E P} \mathbf{4} / \boldsymbol{p b} \mathbf{4}$, we decomposed $\Delta G_{\text {solv }}$ values into $\Delta G_{\text {polar }}$ and $\Delta G_{\text {nonpolar }}$ using $\boldsymbol{E P \mathscr { 2 }}(\boldsymbol{p b} 1-\boldsymbol{p b} 3)$ and $\boldsymbol{E P} \boldsymbol{4}(\boldsymbol{p b} 4)$ to investigate further this question (see Figure 11). The large negative $\Delta G_{\text {solv }}$ values are mainly due to an overestimation of $\Delta G_{\text {polar }}$. Indeed, as one moves from $\boldsymbol{p b} \mathbf{1}$ to $\boldsymbol{p} \boldsymbol{b} \boldsymbol{4}$, the values become less negative and hence, the calculated $\Delta G_{\text {solv }}$ values are typically closer to the ex- 

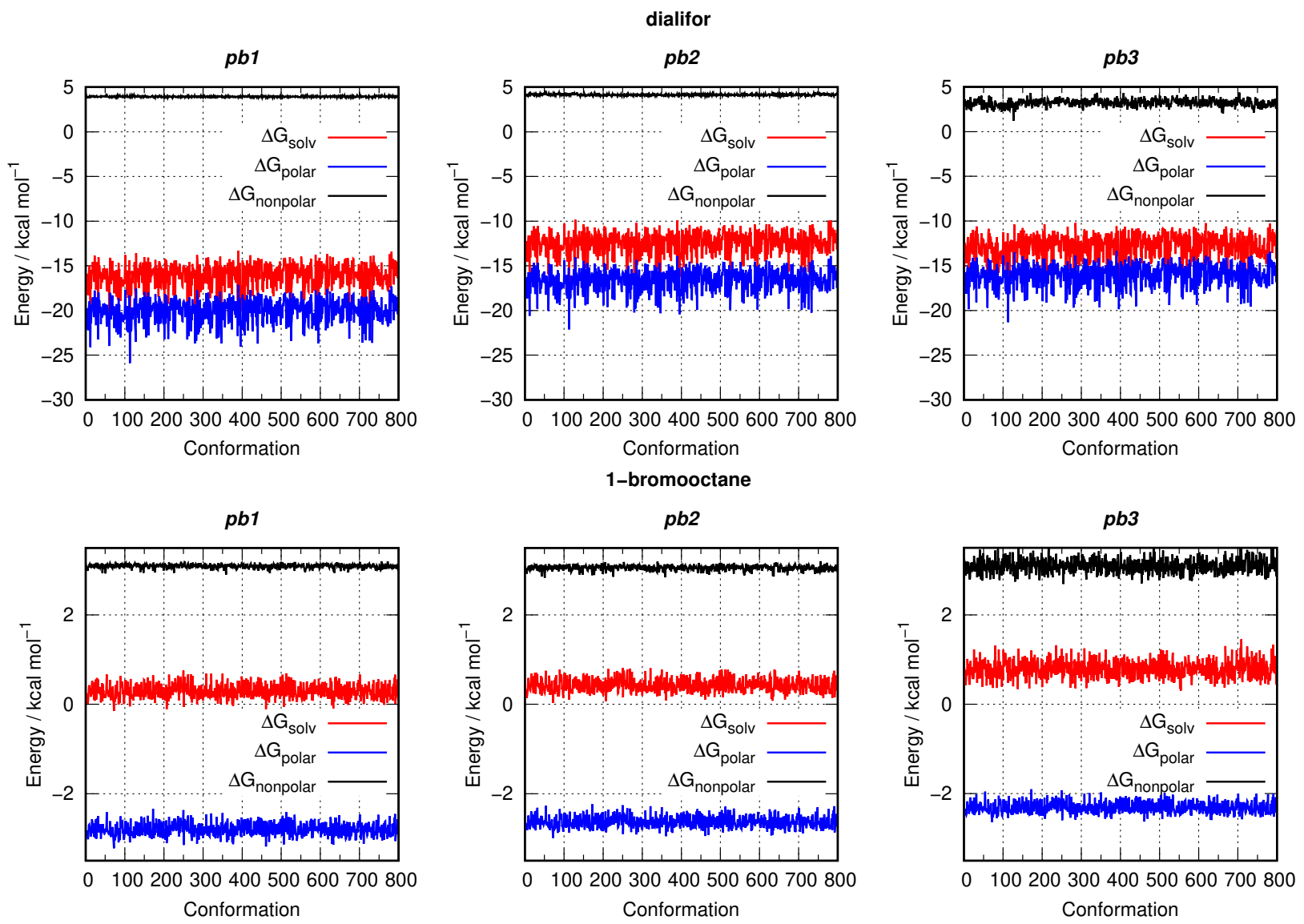

Figure 10: Variation of the calculated $\Delta G_{\text {solv }}$ values along with their components, $\Delta G_{\text {polar }}$ and $\Delta G_{\text {nonpolar }}$, for dialifor and 1-bromooctane using $\boldsymbol{E P} \mathscr{2}$ with optimized radii.

perimental one. Notice, however, the exception in $\boldsymbol{p b} 3$ where in spite of a less negative $\Delta G_{\text {polar }}$ being obtained, the addition of an attractive term renders $\Delta G_{\text {nonpolar }}$ less positive, ultimately leading to a larger deviation when compared with $\boldsymbol{p} \boldsymbol{b} \mathscr{2}$. As mentioned above, using a CHARMM-based EP implementation (EP4) with setup $\boldsymbol{p b} 4$ reduces the deviation to the experimental value in dialifor despite the $\Delta G_{\text {nonpolar }}$ contribution being the smallest. This small positive contribution, quite similar to the one found in $\boldsymbol{p b} \mathbf{3}$ where an attractive term is considered, is balanced by a smaller overestimation of $\Delta G_{\text {polar }}$. These values indicate that the systematic deviation observed for dialifor might arise from a deficient atomic charge assignment owing to the presence of unconventional functional groups (e.g. thiophosphoryl 

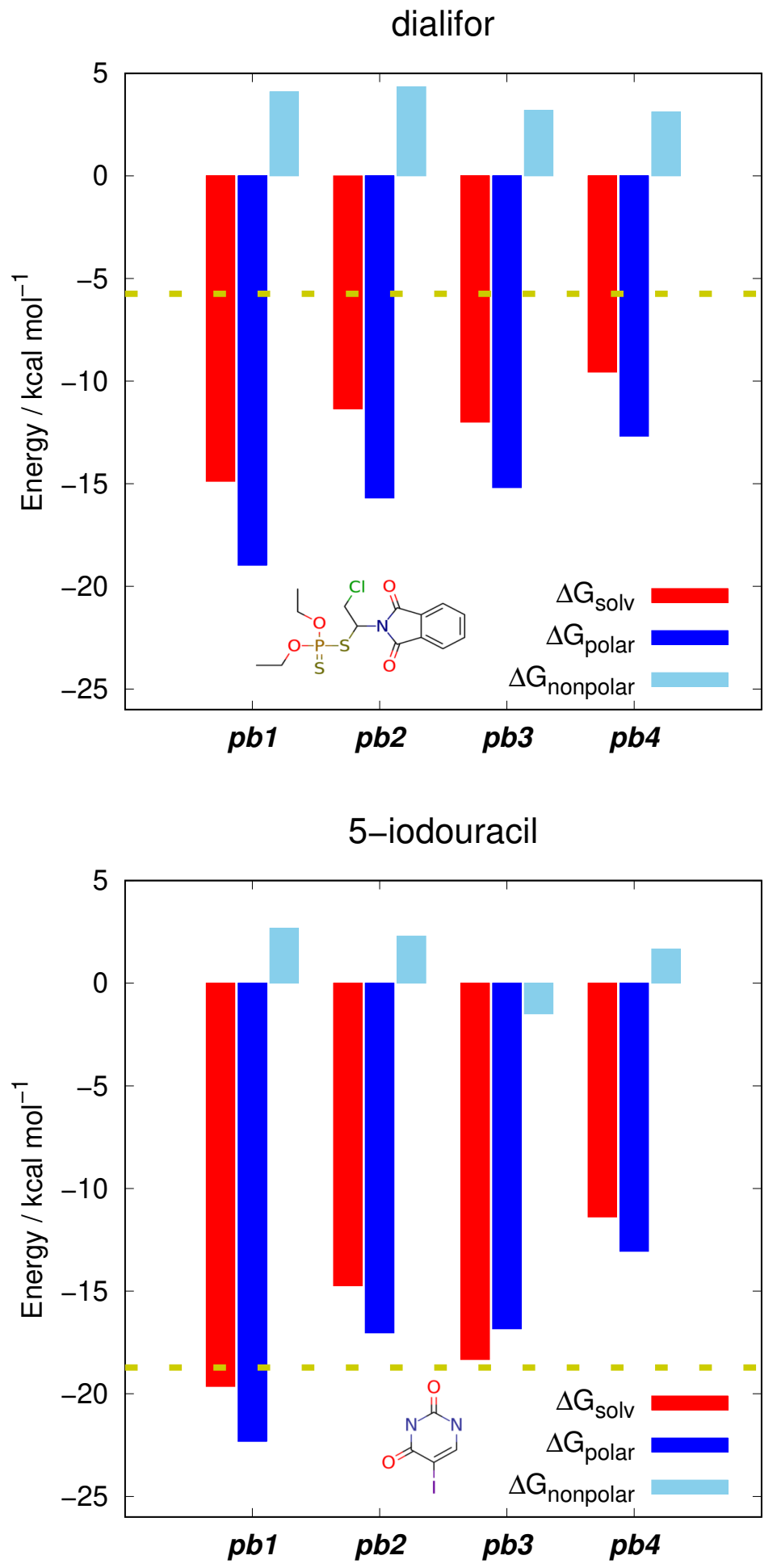

Figure 11: Calculated $\Delta G_{\text {solv }}$ values decomposed into $\Delta G_{\text {polar }}$ and $\Delta G_{\text {nonpolar }}$ for dialifor and 5-iodouracil using $\boldsymbol{E P 2}(\boldsymbol{p b 1}-\boldsymbol{p b} 3)$ and $\boldsymbol{E P} \mathbf{4}(\boldsymbol{p b} 4)$. The dashed horizontal line represents the experimental value 
group) and not from an inaccurate estimate of $\Delta G_{\text {nonpolar }}$ nor the lack of conformational sampling.

In contrast with the observations for dialifor, 5-iodouracil (which was also analyzed earlier using $\left.\boldsymbol{E P} \mathbf{1}^{39}\right)$ is a large outlier for $\boldsymbol{E P} \mathbf{4} / \boldsymbol{p b} \mathbf{4}$. For this molecule the attractive $\Delta G_{\text {dispersion }}$

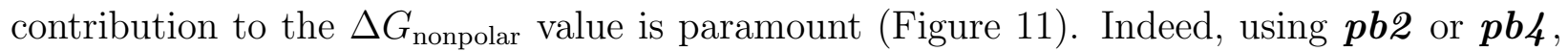
the repulsive nature of $\Delta G_{\text {nonpolar }}$ contributes to enlarge the deviation of $\Delta G_{\text {solv }}$ with respect to the experimental value as $\Delta G_{\text {polar }}$ is not negative enough. With $\boldsymbol{p b} \mathbf{1}$, the $\Delta G_{\text {polar }}$ is slightly too negative but $\Delta G_{\text {nonpolar }}$ is not positive enough to achieve the experimental value. With $\boldsymbol{p b 3}$, the value of $\Delta G_{\text {polar }}$ is less negative but is counterbalanced by an overall negative $\Delta G_{\text {nonpolar }}$ (owing to dispersion), producing a correct estimation of the solvation free energy, in line with previous observations. ${ }^{39}$

\section{Conclusions}

In classical force field methods, the $\sigma$-hole of halogen atoms is often emulated using an off-center point-charge (EP) placed along the $\mathrm{R}-\mathrm{X}$ bond. The inclusion of this EP might introduce artifacts in subsequent Poisson-Boltzmann calculations if the EP is placed at the dielectric interface separating the solute (treated explicitly) and solvent media (treated implicitly), i.e., if the halogen $\mathrm{PB}$ radius is equal to the $\mathrm{X}-\mathrm{EP}$ distance $\left(\mathrm{d}_{\mathrm{X}} \cdots \mathrm{EP}\right) .{ }^{39}$ Moreover, standard halogen PB radii $\left(r_{\text {std }}\right)$ found in the literature can be smaller than $\mathrm{d}_{\mathrm{X}} \cdots \mathrm{EP}$, thus yielding a non-realistic model where the EP is placed in the solvent dielectric. This occurs specially for models placing the off-center point-charge at a fixed distance corresponding

to the Lennard-Jones parameter $R_{\min }{ }^{27}$ as suggested for AMBER/GAFF (herein termed $\boldsymbol{E P 1}$ ), for which a set of compatible halogen PB radii were previously provided. ${ }^{39}$

Since other EP models with diverse $\mathrm{d}_{\mathrm{X}} \ldots \mathrm{EP}$ values and associated charged sets exist, a need for parameters for such models is paramount. In the scope of AMBER/GAFF we selected two different models, one with variable $\mathrm{d}_{\mathrm{X}} \ldots \mathrm{EP}$ values, selected according to the best fit 
to a reference quantum-mechanical electrostatic potential $(\boldsymbol{E P} 2)^{28}$ and another $(\boldsymbol{E P} 3)$ with a fixed $d_{X} \ldots E P$ value and EP charge $\left(\mathrm{qEP}_{\mathrm{EP}}\right){ }^{30}$ By taking a set of 142 halogenated compounds for which experimental $\Delta G_{\text {solv }}$ values are known, we conducted a PB radii optimization using three different PBSA calculation setups. Owing to the variable nature of $\mathrm{d}_{\mathrm{X}} \ldots \mathrm{EP}$ in $\boldsymbol{E P} \boldsymbol{2}$ the variation of the mean absolute errors (MAEs) with the halogen radius shows several error peaks whenever $\mathrm{d}_{\mathrm{X}} \cdots \mathrm{EP}=\mathrm{PB}$ radius for a given compound. Nonetheless, minima could be located and the optimized radii provide MAE values substantially lower than those obtained in standard conditions. Using the best performing PBSA setup which treats the attractive and repulsive interactions separately in the calculation of $\Delta G_{\text {nonpolar }}(\boldsymbol{p b} 3)$, the largest error was c.a. $1 \mathrm{kcal} \mathrm{mol}^{-1}$ for chlorine, the method also providing excellent linear correlations between calculated and experimental values. The optimized radii and respective MAE values are extremly similar to those obtained for $\boldsymbol{E P} 1^{39}$ which is explained by the fact that the distribution of $\mathrm{d}_{\mathrm{X}} \cdots \mathrm{EP}$ values in model $\boldsymbol{E P} \mathscr{2}$ possesses maxima values very close to $R_{\min }$ (where the EP is placed in $\boldsymbol{E P 1}$ ). For $\boldsymbol{E P} 3$ model, a single but broad error peak occurs at $\mathrm{d}_{\mathrm{X} \cdots \mathrm{EP}}=\mathrm{PB}$ radius, with $\boldsymbol{p} \boldsymbol{b} 3$ also being recommended. The optimized radii for this model are different from those obtained earlier with $\boldsymbol{E P} \mathbf{1}$ and $\boldsymbol{E P} 2$ in this work and the errors under optimized conditions are slightly larger but still acceptable (MAEs $<1.3 \mathrm{kcal} \mathrm{mol}^{-1}$ using $p b 3)$.

Aiming at expanding the range of force fields, we also provide the first optimized halogen $\mathrm{PB}$ radii that can be used in the context of the CHARMM General Force Field (CGenFF) which was recently updated to include positively-charged virtual particles to describe halogen bonds $(\boldsymbol{E P} 4) \cdot{ }^{31}$ Since no standard PB radii were available for halogens, we initially took the $R_{\min }$ values as placeholders for $r_{\text {std }}$. Surprisingly, for chlorine, the $R_{\min }$ value is a good approximation for the PB radii as the optimized one is similar $(1.86 \AA$ and $1.84 \AA$, respectively), yielding similar MAE values (c.a. $1.5 \mathrm{kcal} \mathrm{mol}^{-1}$ ), while for bromine and iodine, the $r_{\text {opt }}$ are slightly larger than $r_{\text {std }}$, providing smaller MAE values (c.a. $1.1 \mathrm{kcal} \mathrm{mol}^{-1}$ and $1.4 \mathrm{kcal} \mathrm{mol}^{-1}$, respectively). 
A correct description of halogen bonds is extremely important in the context of computeraided drug design ${ }^{21}$ and models such as $\boldsymbol{E P 1}-\boldsymbol{E P} 4$ are essential to tackle XBs in MM/MD simulations. Although the anisotropy of halogen atoms is better described using an off-center point-charge, one must stress that its inclusion is not strictly justified by an increased accuracy of PBSA calculations as in the absence of EPs, PBSA can also yield similar results under optimized conditions. ${ }^{39}$ The EP inclusion is a condition for the accuracy of MD simulations when halogenated molecules are at play. In this paper we do not discuss nor compare the performance of each one of those models in sampling XBs in (bio)molecular MD simulations, which could depend on the target system. On the contrary, we intend to provide the user with reliable parameters to perform (MM)-PBSA calculations compatible with a prior choice of the off-center point-charge model for MM/MD simulations, which could prove very useful in the context of virtual screening. We must also add that the observed large errors for some molecules may arise from the presence of other elements and complex functional groups and therefore, an optimization of those elements and/or groups is also paramount. In this scope, addressing the anisotropic electronic features in other elements such as sulfur, oxygen, and nitrogen atoms might help in tackling this issue. Moreover, the lack of conformational sampling, specially in the case of long-chain linear haloalkanes, deserves further exploring in future works.

\section{Data and Software Availability}

Halogenated compounds and their respective experimental $\Delta G_{\text {solv }}$ values in water $\left(\Delta G_{\text {hyd }}\right)$ were taken FreeSolv version 0.51 which is freely available (https://github.com/MobleyLab/FreeSolv). CSV files containing the database compound ID, their IUAPC name (or alternative if IUPAC is unavailable) along with experimental and calculated $\Delta G_{\text {solv }}$ values for the selected halogenated compounds are provided in Supporting Information.

In the context of the General AMBER Force Field (GAFF), quantum-mechanical electro- 
static potentials, used as reference to fit RESP charges, were obtained using the commercial software Gaussian 09, Revision A.2 (https://gaussian.com/). A template input file is given in Supporting Information (PDF). RESP charges were obtained the using the antechamber and resp programs which are provided by the AmberTools17 suite that can be obtained free of charge under the GNU General Public License (https://ambermd.org/AmberTools.php).

For EP models featuring the CHARMM General Force Field (CGenFF), atom types and atomic charges were obtained from the Ligand Reader \& Modeler freely available in the CHARMM-GUI platform (http://www.charmm-gui.org/?doc=input/ligandrm) and from the CGenFF server (https://cgenff.umaryland.edu) which is also free upon registration.

All PBSA calculations were performed with the pbsa program from the aforementioned AmberTools17 suite, which is also free of charge. Template input files are also provided in in Supporting Information (PDF).

All MD simulations were performed with GROMACS 2020.04 which can be obtained, free of charge (https://manual.gromacs.org/documentation/2020.4/download.html).

\section{Supporting Information Available}

Supporting Figures and Tables and Input file templates (PDF)

Full list of compounds contained in each subset along with individual experimental and calculated $\Delta G_{\text {solv }}$ values for each EP model / PBSA setup under optimized conditions (CSV)

\section{Acknowledgement}

The authors thank Fundação para a Ciência e a Tecnologia (FCT), Portugal, for the doctoral grant SFRH/BD/146447/2019 (A.F.), and strategic projects UIDB/04046/2020 UIDP/04046/2020 (BioISI) and UID/DTP/04138/2019 (iMed.ULisboa). This work was financed by FCT, Programa Operacional Regional de Lisboa (Lisboa 2020), Portugal 2020, 
FEDER/FN, and the European Union under project number 28455 (LISBOA-01-0145-FEDER028455, PTDC/QUI-QFI/28455/2017).

\section{References}

(1) Jorgensen, W. L. The many roles of computation in drug discovery. Science 2004, 303, $1813-1818$.

(2) Mishra, S. K.; Koča, J. Assessing the performance of MM/PBSA, MM/GBSA, and QM-MM/GBSA approaches on protein/carbohydrate complexes: effect of implicit solvent models, QM methods, and entropic contributions. J. Phys. Chem. B 2018, 122, 8113-8121.

(3) Perlovich, G. L.; Bauer-Brandl, A. Solvation of drugs as a key for understanding partitioning and passive transport exemplified by NSAIDs. Curr. Drug Deliv. 2004, 1, 213-226.

(4) Rizzo, R. C.; Aynechi, T.; Case, D. A.; Kuntz, I. D. Estimation of absolute free energies of hydration using continuum methods: accuracy of partial charge models and optimization of nonpolar contributions. J. Chem. Theory Comput. 2006, 2, 128-139.

(5) Shivakumar, D.; Williams, J.; Wu, Y.; Damm, W.; Shelley, J.; Sherman, W. Prediction of absolute solvation free energies using molecular dynamics free energy perturbation and the OPLS force field. J. Chem. Theory Comput. 2010, 6, 1509-1519.

(6) Christ, C. D.; Fox, T. Accuracy assessment and automation of free energy calculations for drug design. J. Chem. Inf. Model 2014, 54, 108-120.

(7) Cabeza de Vaca, I.; Qian, Y.; Vilseck, J. Z.; Tirado-Rives, J.; Jorgensen, W. L. Enhanced Monte Carlo methods for modeling proteins including computation of absolute free energies of binding. J. Chem. Theory Comput. 2018, 14, 3279-3288. 
(8) Martins, S. A.; Sousa, S. F.; Ramos, M. J.; Fernandes, P. A. Prediction of solvation free energies with thermodynamic integration using the general AMBER force field. J. Chem. Theory Comput. 2014, 10, 3570-3577.

(9) Khavrutskii, I. V.; Wallqvist, A. Improved binding free energy predictions from singlereference thermodynamic integration augmented with hamiltonian replica exchange. J. Chem. Theory Comput. 2011, 7, 3001-3011.

(10) Wang, C.; Nguyen, P. H.; Pham, K.; Huynh, D.; Le, T.-B. N.; Wang, H.; Ren, P.; Luo, R. Calculating protein-ligand binding affinities with MMPBSA: Method and error analysis. J. Comput. Chem. 2016, 37, 2436-2446.

(11) Wang, C.; Greene, D.; Xiao, L.; Qi, R.; Luo, R. Recent Developments and Applications of the MMPBSA Method. Front. Mol. Biosci. 2017, 4, 87.

(12) Clark, T.; Hennemann, M.; Murray, J. S.; Politzer, P. Halogen bonding: the $\sigma$-hole. J. Mol. Model. 2007, 13, 291-296.

(13) Desiraju, G. R.; Ho, P. S.; Kloo, L.; Legon, A. C.; Marquardt, R.; Metrangolo, P.; Politzer, P.; Resnati, G.; Rissanen, K. Definition of the halogen bond (IUPAC Recommendations 2013). Pure Appl. Chem. 2013, 85, 1711-1713.

(14) Cavallo, G.; Metrangolo, P.; Milani, R.; Pilati, T.; Priimagi, A.; Resnati, G.; Terraneo, G. The halogen bond. Chem. Rev. 2016, 116, 2478-2601.

(15) Costa, P. J. The halogen bond: nature and applications. Phys. Sci. Rev. 2017, 2, 20170136.

(16) Brown, A.; Beer, P. D. Halogen bonding anion recognition. Chem. Commun. 2016, 52, 8645-8658.

(17) Nunes, R.; Costa, P. J. Ion-Pair Halogen Bonds in 2-Halo-Functionalized Imidazolium 
Chloride Receptors: Substituent and Solvent Effects. Chem. Asian J. 2017, 12, 586594.

(18) Sutar, R. L.; Huber, S. M. Catalysis of organic reactions through halogen bonding. ACS Catalysis 2019, 9, 9622-9639.

(19) Auffinger, P.; Hays, F. A.; Westhof, E.; Ho, P. S. Halogen bonds in biological molecules. Proc. Natl. Acad. Sci. USA 2004, 101, 16789-16794.

(20) Wilcken, R.; Zimmermann, M. O.; Lange, A.; Joerger, A. C.; Boeckler, F. M. Principles and applications of halogen bonding in medicinal chemistry and chemical biology. $J$. Med. Chem. 2013, 56, 1363-1388.

(21) Costa, P. J.; Nunes, R.; Vila-Viçosa, D. Halogen bonding in halocarbon-protein complexes and computational tools for rational drug design. Expert Opin. Drug Discov. 2019, 14, 805-820.

(22) Nunes, R. S.; Vila-Viçosa, D.; Costa, P. J. Halogen bonding: an underestimated player in membrane-ligand interactions. J. Am. Chem. Soc. 2021, 143, 4253-4267.

(23) Lu, Y.; Shi, T.; Wang, Y.; Yang, H.; Yan, X.; Luo, X.; Jiang, H.; Zhu, W. Halogen bonding-a novel interaction for rational drug design? J. Med. Chem. 2009, 52, 28542862.

(24) Gerebtzoff, G.; Li-Blatter, X.; Fischer, H.; Frentzel, A.; Seelig, A. Halogenation of drugs enhances membrane binding and permeation. ChemBioChem 2004, 5, 676-684.

(25) Ford, M. C.; Ho, P. S. Computational tools to model halogen bonds in medicinal chemistry. J. Med. Chem. 2016, 59, 1655-1670.

(26) Kolář, M. H.; Hobza, P. Computer modeling of halogen bonds and other $\sigma$-hole interactions. Chem. Rev. 2016, 116, 5155-5187. 
(27) Ibrahim, M. A. Molecular mechanical study of halogen bonding in drug discovery. J. Comput. Chem. 2011, 32, 2564-2574.

(28) Rendine, S.; Pieraccini, S.; Forni, A.; Sironi, M. Halogen bonding in ligand-receptor systems in the framework of classical force fields. Phys. Chem. Chem. Phys. 2011, 13, $19508-19516$.

(29) Jorgensen, W. L.; Schyman, P. Treatment of halogen bonding in the OPLS-AA force field: application to potent anti-HIV agents. J. Chem. Theory Comput. 2012, 8, 38953901.

(30) Kolář, M.; Hobza, P. On extension of the current biomolecular empirical force field for the description of halogen bonds. J. Chem. Theory Comput. 2012, 8, 1325-1333.

(31) Gutiérrez, I. S.; Lin, F.-Y.; Vanommeslaeghe, K.; Lemkul, J. A.; Armacost, K. A.; Brooks, C. L.; MacKerell, A. D. Parametrization of halogen bonds in the CHARMM general force field: Improved treatment of ligand-protein interactions. Bioorg. Med. Chem. 2016, 24, 4812-4825.

(32) Franchini, D.; Dapiaggi, F.; Pieraccini, S.; Forni, A.; Sironi, M. Halogen bonding in the framework of classical force fields: The case of chlorine. Chem. Phys. Lett. 2018, 712, 89-94.

(33) Nunes, R.; Vila-Viçosa, D.; Machuqueiro, M.; Costa, P. J. Biomolecular Simulations of Halogen Bonds with a GROMOS Force Field. J. Chem. Theory Comput. 2018, 14, $5383-5392$.

(34) Titov, O. I.; Shulga, D. A.; Palyulin, V. A. Quadrupole correction: from molecular electrostatic potential to free energies of halogen bonding. J. Chem. Theory Comput. 2018, 15, 1159-1167. 
(35) Ibrahim, M. A. A. Molecular mechanical perspective on halogen bonding. J. Mol. Model. 2012, 18, 4625-4638.

(36) Luchi, A. M.; Angelina, E. L.; Andujar, S. A.; Enriz, R. D.; Peruchena, N. M. Halogen bonding in biological context: a computational study of D2 dopamine receptor. J. Phys. Org. Chem. 2016, 29, 645-655.

(37) Ibrahim, M. A. A.; Hasb, A. A. M.; Mekhemer, G. A. H. Role and nature of halogen bonding in inhibitor $\cdot$ receptor complexes for drug discovery: casein kinase-2 (CK2) inhibition as a case study. Theor. Chem. Acc. 2018, 137, 38.

(38) Zhou, Y.; Wang, Y.; Li, P.; Huang, X.-P.; Qi, X.; Du, Y.; Huang, N. Exploring Halogen Bonds in 5-Hydroxytryptamine 2B Receptor-Ligand Interactions. ACS Med. Chem. Lett. 2018, 9, 1019-1024.

(39) Nunes, R.; Vila-Viçosa, D.; Costa, P. J. Tackling Halogenated Species with PBSA: Effect of Emulating the $\sigma$-hole. J. Chem. Theory Comput. 2019, 15, 4241-4251.

(40) Wang, J.; Wolf, R. M.; Caldwell, J. W.; Kollman, P. A.; Case, D. A. Development and testing of a general amber force field. J. Comput. Chem. 2004, 25, 1157-1174.

(41) Bayly, C. I.; Cieplak, P.; Cornell, W. D.; Kollman, P. A. A well-behaved electrostatic potential based method using charge restraints for deriving atomic charges: the RESP model. J. Chem. Phys. 1993, 97, 10269-10280.

(42) Sitkoff, D.; Sharp, K. A.; Honig, B. Accurate calculation of hydration free energies using macroscopic solvent models. J. Phys. Chem. 1994, 98, 1978-1988.

(43) Tsui, V.; Case, D. A. Theory and applications of the generalized Born solvation model in macromolecular simulations. Biopolymers 2000, 56, 275-291.

(44) Onufriev, A. V.; Aguilar, B. Accuracy of continuum electrostatic calculations based 
on three common dielectric boundary definitions. J. Theor. Comput. Chem. 2014, 13, 1440006.

(45) Horton, J.; Allen, A. E.; Dodda, L. S.; Cole, D. J. QUBEKit: Automating the Derivation of Force Field Parameters from Quantum Mechanics. J. Chem. Theory Comput. 2019 ,

(46) Yan, X. C.; Robertson, M. J.; Tirado-Rives, J.; Jorgensen, W. L. Improved Description of Sulfur Charge Anisotropy in OPLS Force Fields: Model Development and Parameterization. J. Phys. Chem. B 2017, 121, 6626-6636.

(47) Roos, K.; Wu, C.; Damm, W.; Reboul, M.; Stevenson, J. M.; Lu, C.; Dahlgren, M. K.; Mondal, S.; Chen, W.; Wang, L.; Abel, R.; Friesner, R. A.; Hardner, E. D. OPLS3e: Extending Force Field Coverage for Drug-Like Small Molecules. J. Chem. Theory Comput. 2019 ,

(48) Mobley, D. L.; Guthrie, J. P. FreeSolv: a database of experimental and calculated hydration free energies, with input files. J. Comput. Aided Mol. Design 2014, 28, 711720.

(49) Mobley, David L. http://www.escholarship.org/uc/item/6sd403pz, 2013; Experimental and Calculated Small Molecule Hydration Free Energies. UC Irvine: Department of Pharmaceutical Sciences, UCI.

(50) Krishnan, R.; Binkley, J. S.; Seeger, R.; Pople, J. A. Self-consistent molecular orbital methods. XX. A basis set for correlated wave functions. J. Chem. Phys. 1980, 72, $650-654$.

(51) McLean, A. D.; Chandler, G. S. Contracted Gaussian basis sets for molecular calculations. I. Second row atoms, $\mathrm{Z}=11-18$. J. Chem. Phys. 1980, 72, 5639-5648. 
(52) Becke, A. D. Density-functional exchange-energy approximation with correct asymptotic behavior. Phys. Rev. A 1988, 38, 3098-3100.

(53) Lee, C.; Yang, W.; Parr, R. G. Development of the Colle-Salvetti correlation-energy formula into a functional of the electron density. Phys. Rev. B 1988, 37, 785-789.

(54) Curtiss, L. A.; McGrath, M. P.; Blaudeau, J.-P.; Davis, N. E.; Binning Jr, R. C.; Radom, L. Extension of Gaussian-2 theory to molecules containing third-row atoms Ga-Kr. J. Chem, Phys. 1995, 103, 6104-6113.

(55) Glukhovtsev, M. N.; Pross, A.; McGrath, M. P.; Radom, L. Extension of Gaussian-2 (G2) theory to bromine-and iodine-containing molecules: Use of effective core potentials. J. Chem. Phys. 1995, 103, 1878-1885.

(56) Frisch, M. J.; Trucks, G. W.; Schlegel, H. B.; Scuseria, G. E.; Robb, M. A.; Cheeseman, J. R.; Scalmani, G.; Barone, V.; Mennucci, B.; Petersson, G. A.; Nakatsuji, H.; Caricato, M.; Li, X.; Hratchian, H. P.; Izmaylov, A. F.; Bloino, J.; Zheng, G.; Sonnenberg, J. L.; Hada, M.; Ehara, M.; Toyota, K.; Fukuda, R.; Hasegawa, J.; Ishida, M.; Nakajima, T.; Honda, Y.; Kitao, O.; Nakai, H.; Vreven, T.; Montgomery, J. A., Jr.; Peralta, J. E.; Ogliaro, F.; Bearpark, M.; Heyd, J. J.; Brothers, E.; Kudin, K. N.; Staroverov, V. N.; Kobayashi, R.; Normand, J.; Raghavachari, K.; Rendell, A.; Burant, J. C.; Iyengar, S. S.; Tomasi, J.; Cossi, M.; Rega, N.; Millam, J. M.; Klene, M.; Knox, J. E.; Cross, J. B.; Bakken, V.; Adamo, C.; Jaramillo, J.; Gomperts, R.; Stratmann, R. E.; Yazyev, O.; Austin, A. J.; Cammi, R.; Pomelli, C.; Ochterski, J. W.; Martin, R. L.; Morokuma, K.; Zakrzewski, V. G.; Voth, G. A.; Salvador, P.; Dannenberg, J. J.; Dapprich, S.; Daniels, A. D.; Farkas, Ö.; Foresman, J. B.; Ortiz, J. V.; Cioslowski, J.; Fox, D. J. Gaussian 09 Revision A.2. Gaussian Inc. Wallingford CT 2009. 
(57) Hariharan, P. C.; Pople, J. A. The influence of polarization functions on molecular orbital hydrogenation energies. Theor. Chim. Acta 1973, 28, 213-222.

(58) Francl, M. M.; Pietro, W. J.; Hehre, W. J.; Binkley, J. S.; Gordon, M. S.; DeFrees, D. J.; Pople, J. A. Self-consistent molecular orbital methods. XXIII. A polarization-type basis set for second-row elements. J. Chem. Phys. 1982, 77, 3654-3665.

(59) Rassolov, V. A.; Ratner, M. A.; Pople, J. A.; Redfern, P. C.; Curtiss, L. A. 6-31G* basis set for third-row atoms. J. Comput. Chem. 2001, 22, 976-984.

(60) Pritchard, B. P.; Altarawy, D.; Didier, B.; Gibson, T. D.; Windus, T. L. New basis set exchange: An open, up-to-date resource for the molecular sciences community. $J$. Chem. Inf. Model. 2019, 59, 4814-4820.

(61) Wang, J.; Wang, W.; Kollman, P. A.; Case, D. A. Automatic atom type and bond type perception in molecular mechanical calculations. J. Mol. Graph. Model. 2006, 25, $247-260$.

(62) Case, D. A.; Cerutti, D. S.; Cheatham III, T. E.; Darden, T. A.; Duke, R. E.; Giese, T. J.; Gohlke, H.; Goetz, A. W.; Greene, D.; Homeyer, N.; Izadi, S.; Kovalenko, A.; Lee, T. S.; LeGrand, S.; Li, P.; Lin, C.; Liu, J.; Luchko, T.; Luo, R.; Mermelstein, D.; Merz, K. M.; Monard, G.; Nguyen, H.; Omelyan, I.; Onufriev, A.; Pan, F.; Qi, R.; Roe, D. R.; Roitberg, A.; Sagui, C.; Simmerling, C. L.; BotelloSmith, W. M.; Swails, J.; Walker, R. C.; Wang, J.; Wolf, R. M.; Wu, X.; Xiao, L.; York, D. M.; Kollman, P. A. AMBER 2017. University of California, San Francisco, 2017.

(63) Koláŕ, M.; Hobza, P.; Bronowska, A. K. Plugging the explicit $\sigma$-holes in molecular docking. Chem. Commun. 2013, 49, 981-983.

(64) Kim, S.; Lee, J.; Jo, S.; Brooks III, C. L.; Lee, H. S.; Im, W. CHARMM-GUI ligand 
reader and modeler for CHARMM force field generation of small molecules. J. Comp. Chem. 2017, 38, 1879-1886.

(65) Jo, S.; Kim, T.; Iyer, V. G.; Im, W. CHARMM-GUI: a web-based graphical user interface for CHARMM. J. Comput. Chem. 2008, 29, 1859-1865.

(66) Vanommeslaeghe, K.; Raman, E. P.; MacKerell Jr, A. D. Automation of the CHARMM General Force Field (CGenFF) II: assignment of bonded parameters and partial atomic charges. J. Chem. Inf. Model. 2012, 52, 3155-3168.

(67) Crowley, M. F.; Williamson, M. J.; Walker, R. C. CHAMBER: Comprehensive support for CHARMM force fields within the AMBER software. Int. J. Quant. Chem. 2009, 109, 3767-3772.

(68) Bordner, A.; Cavasotto, C.; Abagyan, R. Accurate transferable model for water, noctanol, and n-hexadecane solvation free energies. J. Phys. Chem. B 2002, 106, 11009 11015.

(69) Tan, C.; Tan, Y.-H.; Luo, R. Implicit nonpolar solvent models. J. Phys. Chem. B 2007, $111,12263-12274$.

(70) Aleksandrov, A.; Lin, F.-Y.; Roux, B.; MacKerell Jr, A. D. Combining the polarizable Drude force field with a continuum electrostatic Poisson-Boltzmann implicit solvation model. J. Comput. Chem. 2018, 39, 1707-1719.

(71) Nina, M.; Beglov, D.; Roux, B. Atomic radii for continuum electrostatics calculations based on molecular dynamics free energy simulations. J. Phys. Chem. B 1997, 101, $5239-5248$.

(72) Banavali, N. K.; Roux, B. Atomic radii for continuum electrostatics calculations on nucleic acids. J. Phys. Chem. B 2002, 106, 11026-11035. 
(73) Abraham, M. J.; Murtola, T.; Schulz, R.; Páll, S.; Smith, J. C.; Hess, B.; Lindahl, E. GROMACS: High performance molecular simulations through multi-level parallelism from laptops to supercomputers. SoftwareX 2015, 1, 19-25.

(74) Jorgensen, W. L.; Chandrasekhar, J.; Madura, J. D.; Impey, R. W.; Klein, M. L. Comparison of simple potential functions for simulating liquid water. J. Chem. Phys. 1983, 79, 926-935.

(75) Darden, T.; York, D.; Pedersen, L. Particle mesh Ewald: An N log (N) method for Ewald sums in large systems. J. Chem. Phys. 1993, 98, 10089-10092.

(76) Hess, B. P-LINCS: A parallel linear constraint solver for molecular simulation. J. Chem. Theory Comput. 2008, 4, 116-122.

(77) Berendsen, H. J.; Postma, J. v.; van Gunsteren, W. F.; DiNola, A.; Haak, J. R. Molecular dynamics with coupling to an external bath. J. Chem. Phys. 1984, 81, 3684-3690.

(78) Bussi, G.; Donadio, D.; Parrinello, M. Canonical sampling through velocity rescaling. J. Chem. Phys. 2007, 126, 014101.

(79) Parrinello, M.; Rahman, A. Polymorphic transitions in single crystals: A new molecular dynamics method. J. Appl. Phys. 1981, 52, 7182-7190.

(80) Riquelme, M.; Lara, A.; Mobley, D. L.; Verstraelen, T.; Matamala, A. R.; VöhringerMartinez, E. Hydration Free Energies in the FreeSolv Database Calculated with Polarized Iterative Hirshfeld Charges. J. Chem. Inf. Model. 2018, 58, 1779-1797.

(81) Guthrie, J. P. A blind challenge for computational solvation free energies: introduction and overview. J. Phys. Chem. B 2009, 113, 4501-4507.

(82) The legacy version version of CGenFF, which does not add an EP to halogen atoms, did not support the following chlorinated molecules owing mostly to the SP bond not being supported: mobley_2996632 (chloroform), mobley_6359135 (car- 
bon tetrachloride), mobley_8311321 (chloro-difluoro-methane), mobley_1849020, (4chlorophenyl)sulfanylmethylsulfanyl-diethoxy-thioxo- $\lambda^{5}$-phosphane), mobley_2518989 (dialifor), and mobley_7326706 (chlorpyrifos).

(83) Kongsted, J.; Söderhjelm, P.; Ryde, U. How accurate are continuum solvation models for drug-like molecules? J. Comput. Aided Mol. Des 2009, 23, 395-409.

(84) Luukkonen, S.; Belloni, L.; Borgis, D.; Levesque, M. Predicting Hydration Free Energies of the FreeSolv Database of Drug-like Molecules with Molecular Density Functional Theory. J. Chem. Inf. Model. 2020, 60, 3558-3565.

(85) Matos, G. D. R.; Kyu, D. Y.; Loeffler, H. H.; Chodera, J. D.; Shirts, M. R.; Mobley, D. L. Approaches for calculating solvation free energies and enthalpies demonstrated with an update of the FreeSolv database. J. Chem. Eng. Data 2017, 62, 1559-1569. 
Graphical TOC Entry

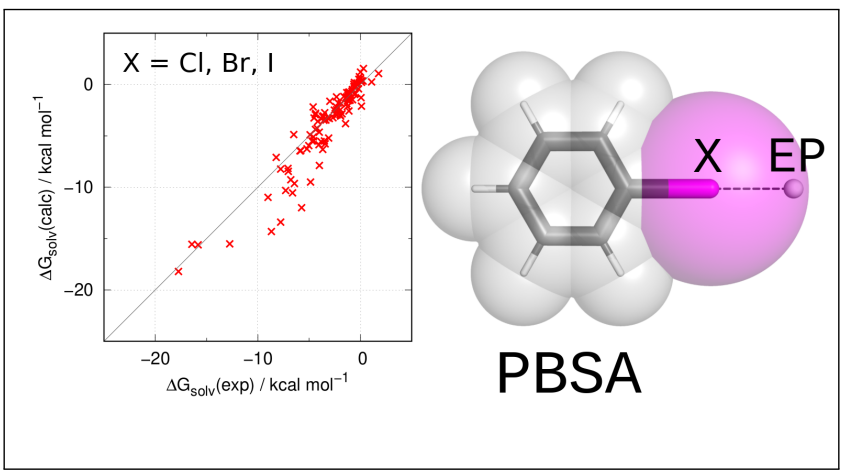

